\title{
Atención Materno Infantil Estudio Comparativo Enero-Diciembre 1980
}

\author{
Dres.: Luzmila Arango de Calderón, Carlos Olaya*, Javier Muñoz**, Francisco Pardo*** \\ Sonia Pazmiño de Osorio, Francisco Pérez, Carlos Osorio Torres, Jaime Barreto, \\ Gustavo Gómez**** y Sonia Pazmiño de Osorio*****.

\section{CONGRESO NACIONAL DE OBSTETRICIA Y GINECOLOGIA CARTAGENA, DICIEMBRE 1981}

\section{AGRADECIMIENTOS}

\begin{abstract}
A todas las personas, médicos internos, enfermeras, auxiliares, promotoras que colaboraron en la investigación, sin las cuales no hubiera sido posible realizarla
\end{abstract}

Resumen general

Se encuestaron 12.218 familias que corresponden a una población de 65.420

* Sociedad de Obstetricia y Ginecología del Tolima.

* Sociedad de Obstetricia y Ginecología de Nariño.

*** Sociedad Colombiana de Obstetricia y Ginecología de Bogotá.

Sociedad Vallecaucana de Obstetricia y Ginecología.

Coordinación general: Sociedad Va. llecaucana. personas en las ciudades de Cali, Bogotá, Ibagué, Pasto, lo que ha permitido profundizar en el estudio sobre la atención materno infantil en estas ciudades, cumpliendo los objetivos del estudio.

Se encontraron diferencias claras en los indicadores de salud de las ciudades participantes.

En el departamento del Valle, donde el estudio se realizó en todas las regionales en que se encueritra subdividido, también se hallaron diferencias entre ellas. 
Se ha logrado obtener indicadores actualizados $y$ por primera vez se obtienen tasas de Mortinatalidad y Mortalidad perinatal. La Mortalidad neonatal en Cali, 13.9, es similar a los estudios de California (E. U.). En Ibagué, 19.7, es mayor y en Pasto, 30.6, el doble.

Se plantea la necesidad de estudios que aclaren las diferencias anotadas.

\section{CAPITULO I}

\section{Concepto de salud y enfermedad}

La salud y la enfermedad no constituyen fenómenos opuestos, sino diferentes grados de adaptación del organismo frente al ambiente en que vive.

La salud es, por tanto, el equilibrio que resulta de la interacción entre el hombre y los variables requerimientos del medio al que está íntimamente vinculado; al romperse este equilibrio se pasa al estado de enfermedad.

Salud y enfermedad, dos grados extremos en la variación biológica, son la resultante del éxito o del fracaso del organismo para adaptarse física y mentalmente, a las condiciones variables del ambiente.

Dos tipos de factores influyen el proceso de adaptación: imperfecciones hereditarias congénitas o biológicas y factores hostiles del ambiente externo ante los cuales el organismo tiene dificultades para adaptarse. Factores estos que se conjugan y son claramente palpables en la atención de la madre y el niño.

Por lo tanto, un individuo sano es aquel que muestra armonía física y men- tal $y$ adaptación al ambiente físico $y$ social $y$ a sus variaciones, de forma tal que puede contribuír al bienestar de la sociedad de acuerdo a su habilidad (1).

\section{FACTORES DEL MEDIO EXTERNO}

\section{SANEAMIENTO AMBIENTAL DE LAS AMERICAS (2)}

\section{Abastecimiento de agua}

En 1977, unos 155 millones de habitantes de las zonas urbanas latinoamericanas $178 \%$ de la población urbana total), disponían de servicio de agua potable. En Colombia el $73.9 \%$ (1979) (21).

En las zonas rurales sólo el 34\% de los habitantes disponían de ese servicio. En Colombia sólo el 13\% (1979) (21).

\section{Sistema de alcantarillados}

Hacia 1977 sólo 10 de 25 países latinoamericanos ofrecieron servicio de alcantarillado a más del $45 \%$ de los habitantes urbanos. En Colombia el $61.7 \%$.

El medio rural presenta una situación menos favorable. De 31 países sobre los que se disponía de datos en 1977 únicamente Ecuador, Panamá y la República Dominicana ofrecieron servicios de alcantarillado a más del $10 \%$ de la población rural. En Colombia sólo el $7.1 \%$.

\section{Disponibilidad de alimentos}

La disponibilidad de alimentos varía considerablemente en los distintos países de la región y dentro de ellos, según las distintas áreas geográficas.

Las cifras oscilan entre 1900 calorías disponibles (EI Salvador y Haití) y 3000 calorías (Argentina y Uruguay). 
Cuadro No. 1

\section{DISPONIBILIDAD Y CONSUMO DE CALORIAS Y PROTEINAS POR HABITANTE POR DIA, AMERICAS, PAISES SELECCIONADOS 1974 O ULTIMO AÑO DISPONIBLE (3)}

\begin{tabular}{|l|c|c|c|c|}
\hline \multirow{2}{*}{ País } & \multicolumn{2}{|c|}{ Calorías } & \multicolumn{2}{c|}{ Prote ína (Gramos) } \\
\cline { 2 - 5 } & Disponibilidad & Consumo & Disponibilidad & Consumo \\
\hline Argentina & 3.036 & & 98.4 & \\
Brasil & 2.613 & 2.640 & 64.5 & 77.0 \\
Colombia & 2.160 & 1.812 & 48.1 & 46.1 \\
Costa Rica & 2.344 & 1.961 & 61.3 & 55.7 \\
Méjico & 2.660 & 2.077 & 67.1 & \\
Panamá & 2.429 & 2.091 & 65.4 & 62.2 \\
Uruguay & 3.105 & 3.259 & 108.2 & 116.0 \\
\hline
\end{tabular}

$\begin{array}{lr}\bar{X} \text { Adultos/dia }= & 3000 \text { calorías } \\ \bar{X} \text { Adultos/dia }= & 68 \mathrm{~g} . \text { proteínas }\end{array}$

Se ha encontrado que la población de América Latina tiene una alimentación deficiente en energía, proteína, vitamina $A$, riboflavina, hierro, folatos $y$ yodo y especificamente en mujeres embarazadas se ha comprobado carencias alimenticias continuadas, especialmente de vitamina $A$, energia, riboflavina y hierro.

La carencia de folatos se hace más evi. dente cuando se administra hierro a mu jeres embarazadas. Se ha observado, que los sueros del $58 \%$ de una muestra de mujeres embarazadas de bajo nivel socio económico eran pobres en folatos.

Esto merece especial atención a causa de la acumulación de datos indicativos de que la carencia de folatos durante el embarazo afecta negativamente el desarrollo fetal.
Todo parece indicar que el estado nutricional del feto refleja en muchos aspectos carencias de la madre. Por lo tanto, la nutrición materna tiene gran importancia en Salud Pública porqueafecta no sólo a las madres sino a los niños por nacer (4).

El embarazo y la lactancia acrecientan la necesidad de nutrientes en 200 calorías y 12 grs. de proteína y 800 calorias y 18 grs., respectivamente (22).

El factor que limita con mayor frecuencia la capacidad de adquirir los alimentos necesarios para cubrir las recomendaciones energéticas y de nutrientes, es el bajo ingreso de sectores importantes de la población.

Se ha establecido relación entre la ganancia de peso de la embarazada y el 
peso del recién nacido; entre menor ganancia de peso durante el embarazo mayor riesgo de bajo peso al nacer con mayor morbilidad posterior.

La experiencia tanto clínica como de Salud Pública sugiere, que con excepción de los casos de patología especial, el al to riesgo de dar a luz niños con bajo peso al nacer puede reducirse notablemente aplicando medidas orientadas a mejorar el estado nutricional y de salud de la madre embarazada. (5).

\section{Situación de salud de}

\section{la adolescente (6)}

La salud del adolescente en general está fuertemente condicionado por el desarrollo de las etapas previas. Además, el proceso de maduración que experimenta el joven influye en las nuevas enfermedades y puede transferir a las etapas posteriores de la vida los trastornos que no han podido ser superados y aún pueden comprometer el futuro de su descendencia.

El adolescente presenta proporcionalmente una baja mortalidad global, lo que limita en parte el análisis de la situación de salud. En cambio las tasas de mortalidad por causas muestran la incidencia de factores que requieren una clara definición intersectorial de atención a este grupo.

Dadas las variaciones individuales con que se presentan los cambios que se producen entre los 10 y 19 años, es evidente que los adolescentes forman un

Cuadro No. 2

\section{PRINCIPALES CAUSAS DE DEFUNCION EN MUJERES DE 15 - 19 AÑOS}

\begin{tabular}{|c|c|c|c|c|c|}
\hline \multicolumn{2}{|c|}{ America del Norte } & \multicolumn{2}{c|}{ Mesoamérica } & \multicolumn{2}{c|}{ América del Sur } \\
\hline Causa & $\%$ \% & Causa & $\%$ & Causa & \% \\
\hline 1 & 48.5 & 1 & 14.1 & 1 & 19.0 \\
2 & 8.8 & 6 & 8.2 & 12 & 9.0 \\
7 & 8.6 & 12 & 8.1 & 10 & 6.7 \\
10 & 5.6 & 4 & 6.2 & 6 & 6.2 \\
6 & 2.8 & 5 & 5.8 & 4 & 5.7 \\
11 & 25.6 & 11 & 57.6 & 11 & 53.4 \\
\hline Total & 100.0 & Total & 100.0 & Total & 100.0 \\
\hline
\end{tabular}

1. Accidentes

2. Neoplasias malignas

3. Anomalías congénitas

4. Influencia y neumonía

5. Enteritis y otras enfermedades

6. Enfermedades cardíacas

7. Homicidios y operaciones de guerra
8. Anemias.

9. Tuberculosis

10. Suicidios

11. Otras enfermedades

12. Complicaciones del embarazo parto y puerperio 
grupo heterogéneo desde el punto de vista de la salud.

Los datos analizados muestran la importancia de las complicaciones del embarazo, parto y puerperio como causa de muerte en el grupo de mujeres de 15 a 19 años. Siendo la tercera y segunda causa de muerte de Mesoamérica y América del Sur, respectivamente, después de los accidentes.

El orden de importancia y el porcentaje de defunciones por esta patología en algunos países de las américas, se presentan en el siguiente cuadro de acuerdo a los datos del último año disponible.

En tres países fué la primera causa de muerte, en cuatro fué la segunda; Colombia se encuentra en este grupo. En seis, fué la tercera y en todos los países incluídos, con excepción de los Estados Unidos, se contó entre las cinco primeras causas.

\section{Cuadro No. 3}

ORDEN DE IMPORTANCIA Y PORCENTAJE DE DEFUNCIONES POR COMPLICACIONES DEL EMBARAZO, DEL PARTO Y DEL PUERPERIO EN LA POBLACION FEMENINA DE 15 A 19 AÑOS, EN 19 PAISES DE LAS AMERICAS ALREDEDOR DE 1975

\begin{tabular}{|l|c|c|r|}
\hline \multicolumn{1}{|c|}{ Pais } & Año & $\begin{array}{c}\text { Orde de } \\
\text { Importancia }\end{array}$ & \multicolumn{1}{c|}{$\%$} \\
\hline Argentina & 1970 & III & 8.1 \\
Chile & 1975 & II & 8.6 \\
Colombia & 1975 & II & 9.6 \\
Costa Rica & 1975 & III & 10.3 \\
Paraguay & 1975 & $\mathrm{I}$ & 16.2 \\
Venezuela & 1975 & $\mathrm{~V}$ & 4.6 \\
\hline
\end{tabular}

La magnitud de este problema se manifiesta en estas cifras, que requieren ser debidamente consideradas a pesar del sesgo ya conocido que pueden presentar.

Entonces, la salud es un producto intersectorial que depende del desarrollo global de una región.

Es por esto que a través de algunos indicadores como los de vivienda, educación, morbilidad y mortalidad, permiten revelar las diferencias en "calidad de vida" de los países desarrollados y los así denominados sub-desarrollados (según último año disponible).

\section{Cuadro No. 4}

\section{INDICADORES INDIRECTOS DEL ESTADO NUTRICIONAL MORTALIDAD ( 3.7 )}

\begin{tabular}{|l|c|c|c|}
\hline \multicolumn{1}{|c|}{ País } & Materna & $\begin{array}{c}\text { Neonatal } \\
<28 \text { días }\end{array}$ & $\begin{array}{c}\text { Infantil } \\
<1 \text { año }\end{array}$ \\
\hline Estados Unidos & 0.24 & 14.2 & 18.5 \\
Colombia & 3.00 & 25.1 & 92.7 \\
Chile & 1.63 & 29.0 & 71.1 \\
Ecuador & 1.09 & 23.0 & 75.8 \\
Salvador & 1.18 & 18.4 & 58.5 \\
Paraguay & 4.16 & - & 84.0 \\
\hline
\end{tabular}

A pesar del sub-registro permanente que no permite aflorar la real magnitud del problema, se ha detectado que la situación de la salud Materno-Infantil en América Latina y el Caribe, se caracteriza por la alta prevalencia de los daños reductibles.

Los estudios sobre el período perinatal, desde la 28a. semana de gestación hasta el séptimo día de vida postnatal, muestran que este es crítico para la salud de la madre y el recién nacido, por lo cual debe asignarse una al ta prioridad a los cuidados maternos en los primeros 
estadios del embarazo y aún antes de la concepción. En éste período de la vida, los indicadores más importantes utilizados son la mortalidad materna y la mortalidad perinatal.

Es notable la escasez de datos sobre la mortalidad perinatal en América Latina y específicamente en nuestro país se desconoce su magnitud.

El análisis de la.mortalidad neonatal muestra diferencia notable en los distintos lugares en que se realizaron los proyectos de la investigación interamericana de mortalidad en la niñez (8).

Así, en los estudios de California (Estados Unidos) y Sherbrooke (Canadá), las tasas por 1000 recién nacidos vivos, fueron de 12.7 y 13.5 respectivamente, mientras que en la Provincia de San Juán (Argentina) y Recife (Brasil), las tasas alcanzaron valores de 38.8 y 35.3 respectivamente dentro del grupo de edad de $0-28$ días. Se encontró que las causas perinatales desempeñaban un papel predominante en la mortalidad en la primera semana de vida (0-7 días).

Los resultados de esta investigación mostraron que la inmadurez constituye un problema muy grave en muchos sectores de América Latina. En 9 de los 15 proyectos, más del $60 \%$ de las defunciones neonatales, correspondió a niños con esta definición.

La atención de la Salud Materno Infantil constituye una de las áreas de mayor significado en la región de las Américas, tanto por la magnitud del Universo como por èl tipo de problemas. Es el caso de la mujer trabajadora cuyo contacto con algunas sustancias químicas puede afectar su progenie y que hasta el momento es un campo poco investigado (9).

En el primer taller internacional sobre metodología para la normalización ma- terno-infantil efectuado en la ciudad de la Habana, del 10 al 23 de Noviembre de 1979, con el patrocinio del Ministerio de Salud Pública de Cuba y la organización Panamericana de Salud (10), después de revisar los programas de atención materno-infantil de los países participantes, Méjico, Nicaragua, Panamá, Perú, República Dominicana, Venezuela y Cuba, se puntualizó que existe un déficit cuantitativo expresado por la baja cobertura de la población materno-infantil.

Esta situación se consideró que está determinada, entre otros factores, por los siguientes:

1. Inaccesibilidad de la población a los servicios de salud, no sólo por la inapropiada distribución geográfica de establecimientos, sino por la presencia de otros factores como el analfabetismo, privación cultural en poblaciones urbanas, marginadas y rurales. Es claro que existen en nuestro país regiones donde el acceso a la atención médica está muy limitado para la mayoría de la población y sólo pequeños sectores tienen acceso a servicios de elevada calidad.

2. Multiplicidad de instituciones públicas y privadas, que participan en la prestación de servicios y la ausencia o incipiente coordinación de los mismos.

3. Escasez general de recursos humanos.

No sólo en el número sino en la concentración de éstos, con mayor capacitación en las áreas urbanas y la desprotección en las áreas rurales.

4. Inadecuado sistema de información debido a la falta de confiabilidad en los datos y/o ausencia del los mismos.

Por lo tanto, se deduce que los servicios de salud analizados generan una oferta no sistematizada en cantidad y calidad y sólo responden en forma parcial a la demanda. 


\section{SOLUCIONES PLANTEADAS "SALUD PARA TODOS EN EL AÑO 2.000" (11)}

Se hace énfasis entonces en el tercer mundo, para desarrollar sistemas y mecanismos que hagan viable el contacto con el sistema de salud a millones de seres humanos que actualmente se encuentran desprotegidos.

Se hace necesario desarrollar niveles de atención que vayan de lo más simple a lo más complejo, de tal forma que se ajusten a los recursos de atención para dar a toda la población un cuidado primario, que sea capaz de detectar los casos de mayor complejidad para ser remitidos a niveles superiores de satisfacción.

Para llevar a cabo lo anterior surgen diversos sistemas de calificación de la seriedad de la morbilidad, conocida por todos como calificación de riesgo, que se promueve y se emplea en general en medicina pero que ha determinado una constante investigación en el área obstétrica, sin que eso quiera decir que se ha encontrado un patrón o sistema igualmente aplicable a todos los países; por el contrario, se plantea la necesidad de más investigaciones y determinación de sistemas por regiones.

\section{REPERCUSIONES DE LOS FACTORES DE RIESGO EN LA GESTION (11)}

El principal objetivo del método basado en los factores de riesgo consiste en utilizar en forma óptima los recursos existentes, en beneficio de la mayoría de la población. Trata de garantizar a todos una atención mínima, facilitando directrices que permitan destinar prioritariamente los limitados recursos existentes a quienes más los necesiten.

\section{Empleo no planificado de los recursos}

La figura muestra una forma corriente de utilización de los recursos por una parte de la población, en donde no son precisamente los de mayor riesgo los que más lo utilizan.

Se produce un mal empleo de los recursos; el personal muy capacitado no utiliza sus conocimientos especializados, mientras que quedan al márgen muchos que necesitan la aplicación de tales conocimientos.

"La estrategia de intervención en función del riesgo puede definirse como: política social y sanitaria de intervención activa, basada en datos válidos relativos a los costos, los recursos, los riesgos de enfermedad y la eficacia de las distintas medidas en diferentes lugares.

Una clasificación de los riesgos, basada en los conocimientos adquiridos de casos individuales y en los estudios epidemiológicos locales, permite mejorar la planificación, la programación y la administración de los servicios de salud de la madre y el niño.

En la aplicación de estas clasificaciones, sin embargo, se debe ser muy cuidadoso. En Cuba, por ejemplo, a partir de 1975, después de tener una cobertura total, comenzó a utilizarse en todo el país una clasificación de alto riesgo obstétrico basada en un sistema de puntuación aditivo, modificación de un sistema de puntuación desarrollado en Argentina. La experiencia con este nuevo sistema ha demostrado que aunque su sensibilidad es relativamente alta, su valor predictivo es bajo y su aplicabilidad muy baja ya que el $82 \%$ de las mujeres son consideradas en riesgo. (12).

En resumen, una clasificación de riesgo obstétrico para incidir en la morbi- 
mortalidad materno-infantil, debe ir directamente relacionada con los recursos asignados al sector salud $\mathrm{y}$ con el establecimiento de estrategias de intervención, fuera del sector de la asistencia sanitaria individual.

Entre esas actividades figuran por ejemplo, la higiene del medio, el desarrollo de la colectividad, la instrucción de los adultos y la rotación de cultivos, actividades destinadas a aumentar el nivel de vida y reducir numerosos factores de riesgo.

Para establecer la estrategia de riesgo es necesario establecer una línea de base con el diagnóstico de salud por región, que permita adecuar los criterios de utilización de servicios y los recursos en salud, y una evaluación objetiva que permita observar las modificaciones en la población donde se aplica la estrategia.

\section{Los registros en Colombia}

Considerando que la población materno-infantil en el país corresponde aproximadamente al $70 \%$ de toda la población, no existen registros que permitan el diseño adecuado de los planes preventivos asistenciales para su atención.

Las estadísticas nacionales adolecen de serias limitaciones que las hacen poco confiables, especialmente por sub-registros de la morbi-mortalidad (13-8), y la dificultad en los estimativos de la población a riesgo; recuérdese que el último censo del país tuvo serias fallas y la última encuesta de morbilidad y recursos humanos conocida para el país, fué realizada hace 16 años (1964) (14); por lo tanto, se dificulta la obtención de tasas reales y oportunas que permitan analizar $y$ evaluar la situación maternoinfantil. Es notable además, cómo la evaluación de la asistencia oficial brindada a la madre y el niño, así como su planeación anual se ha cifrado en pará- metros numéricos de consulta o procedimiento, que no reflejan los cambios que pueden suscitarse en la población (15-16).

Al no disponer de parámetros básicos, claros y definidos, se hace muy difícil su evaluación.

En el boletín epidemiólogo Nacional sobre Mortalidad Materno-Infantil de VII/77 (17), donde se aclara la imposibilidad de obtener la mortinatalidad y mortalidad perinatal, nos sorprende el aumento de las dos primeras que contradice las curvas descendentes proyectadas para nuestro país desde 1965. Es necesario hacer notar que el porcentaje del presupuesto nacional de salud, que en 1974 era de $11.36 \%$, ha descendido paulatinamente $\mathrm{y}$ en 1979 corresponde sólo al $7.75 \%$ (23).

Se hace entonces necesario estudiar los cambios producidos y las posibles causales de los mismos, pues disponemos de muy escasas investigaciones que muestren las características de la población que pueden estar relacionadas con los cambios de tasas (18-19-20).

Es por lo tanto una necesidad inaplazable establecer estudios que permitan obtener parámetros que reflejen mejor el estado de salud de la población, para una adecuada planeación y prestación de servicios.

\section{CAPITULO II}

\section{INTRODUCCION}

Desde hace algunos años en los Congresos de Obstetricia se ha mostrado interés en conocer más a fondo la situación de la salud de la madre y el niño en el país; en el último Congreso realiza- 
do en Bogotá en Diciembre de 1979, se decide aunar los esfuerzos de las Sociedades Científicas para profundizar su investigación.

Por lo tanto, se propone este trabajo que cuenta desde ya entre sus logros positivos la vinculación de algunas Sociedades Científicas al sector oficial, en el estudio de los problemas nacionales.

\section{Plan trazado}

Investigar el estado de Salud MaternoInfantil en algunas regiones del país, a través de interrogatorio directo de la población, por medio de encuestas casa a casa.

El período a investigar comprende de Enero a Diciembre de 1980.

El plan presenta cuatro limitantes:

1. La participación voluntaria de las Sociedades Científicas en el estudio, excluyéndose por lo tanto las regiones en donde no se aceptó.

2. En Bogotá se incluyó únicamente un sector de programa materno-infantil especial.

3. Presupuestal. Al disponer básicamente de los escasos recursos propios de las Sociedades

4. La muestra de población involucra en su gran mayoría clase baja.

\section{SOCIEDADES PARTICIPANTES}

1. Sociedad Colombiana, Bogotá. Area: Barrios donde funciona el programa P.A.M.I., hace algunos años. Coordinador: Dr. Francisco Pardo.
2. Sociedad Tolimense, Ibagué.

Area: Ibagué, muestra representativa. Coordinador: Dr. Carlos Olaya. Dra. Luzmila Arango de Calderón.

3. Sociedad Nariñense, Pasto.

Area: Barrio, muestra representativa. Coordinador: Dr. Javier Muñoz.

4. Sociedad Vallecaucana de Obstetricia y Ginecología, Cali.

Coordinadora: Dra. Sonia Pazmiño de Osorio.

La investigación se presentó a las directivas de los servicios de Salud oficiales, encontrándose gran acogida $\mathrm{y}$ aceptación en Ibagué, Cali, Valle y Pasto.

\section{Objetivo general}

Profundizar en el conocimiento del estado de la salud de la madre y el niño en las regiones vinculadas.

\section{Específicos:}

1. Establecer parámetros reales que midan la calidad de la atención materno-infantil.

2. Iniciar la vinculación de las Sociedades Científicas al estudio de los problemas nacionales que comprometan la especialidad $y$ en el planteamiento de sus soluciones.

\section{Antecedentes}

Se envió a todas las Sociedades del país un protocolo con las bases mínimas del estudio, un cuestionario inicial para las visitas a la población, casa a casa, y una carta de información con tres alternativas de estudio, el 31 de Mayo de 1980.

Se realizó una reunión en Cali el día 13 de Septiembre de 1980, en donde las 
Sociedades enviaron sus representantes y el tema escogido para participar. así:

Se escogieron dos temas para el relato

Diagnóstico perinatal con Antioquia y Valle de Coordinadores, y Atención Materno-Infantil con Valle de Coordinador.

Decidieron trabajar en el segundo tema Bogotá, Pasto, Ibagué y Valle.

\section{Metodología}

Delimitado el sector de la población donde se realizaría la investigación, se diseñó, planificó y tomó una muestra representativa que permitiera inferencia estadística. (Anexo No. 1).

Una vez seleccionadas las viviendas a través de mapas actualizados de la zona, se instruyó al personal que llevaría a cabo las encuestas casa a casa.

Al personal se le distribuyó el sector correspondiente y la supervisión y revisión de formularios, se realizó de parte de los investigadores diaria o semanalmente de acuerdo a la accesibilidad del área. Se tomó el $10 \%$ de las encuestas realizadas para una segunda visita e interrogatorio para evaluar la calidad de la información, asegurando la calidad del dato.

Finalmente las encuestas fueron procesadas para posteriormente practicar el análisis estadístico correspondiente.

Bogotá y Pasto realizaron la investiydción en base del protocolo, cuestionario y conversaciones para unificar criterios, con la coordinadora general.

Ibagué fué visitado en su Sede y empleó igual papelería que el Valle.

Cada región nombró su coordinador quien directamente supervisó el trabajo $y$ es su responsable.

Finalmente se hizo una reunión de los investigadores para discusión y análisis de resultados.

\section{EI instrumento}

Está diseñado para medir los siguientes parámetros.

1. Distribución de la población.

2. Grado de escolaridad.

3. Tasa de natalidad.

4. Tasa de aborto.

5. Tasa de mortinatos.

6. Tasa de mortalidad neonatal.

7. Tasa de mortalidad perinatal.

8. Morbilidad durante el embarazo.

9. Utilización y accesibilidad de servicios institucionales para control prenatal, aborto y parto, control del niño sano y vacunación.

10. Tasa de mortalidad infantil.

Anexo No. 2 - Formulario y Tabla. de Códigos.

\section{CAPITULO III}

\section{RESULTADOS COMPARATIVOS \\ PARA CALI, IBAGUE, PASTO Y P.A.M.I. (BOGOTA)}

Se comparan los resultados de las encuestas realizadas en las ciudades de Cali, Ibagué, Pasto y Bogotá (P.A.M.I.). 
Tabla No. 1

\section{FAMILIAS Y POBLACION EN LA MUESTRA PARA CADA CIUDAD}

\begin{tabular}{|c|c|c|c|}
\hline Ciudad & \begin{tabular}{|c|} 
Total \\
Familias
\end{tabular} & $\begin{array}{c}\text { Población } \\
\text { Total }\end{array}$ & $\bar{x} p \times F$ \\
\hline Cali & 7.551 & 39.092 & 5.2 \\
\hline Ibagué & 2.867 & 15.412 & 5.4 \\
\hline $\begin{array}{l}\text { Bogotá } \\
\text { (P.A.M.I.) }\end{array}$ & 970 & 5.889 & 6.1 \\
\hline Pasto & 830 & 5.027 & 6.1 \\
\hline Total: & 12.218 & 65.420 & \\
\hline
\end{tabular}

Tabla No. 1. Se encuestaron en total 65.420 personas que corresponden a 12.218 familias con un promedio de miembros por familia en Cali e Ibagué de 5 y en el P.A.M.I. y Pasto de 6 .

Tabla No. 2

\section{DISTRIBUCION PORCENTUAL POR GRUPOS DE EDAD}

\begin{tabular}{|r|r|r|c|r|}
\hline $\begin{array}{c}\text { Edad } \\
\text { (Años) }\end{array}$ & Cali & Ibagué & $\begin{array}{c}\text { Bogotá } \\
\text { (P.A.M.I.) }\end{array}$ & Pasto \\
\hline 1 & 2.8 & 3.0 & 2.9 & 4.6 \\
$1-14$ & 36.1 & 39.3 & 35.9 & 39.9 \\
$15-44$ & 48.0 & 45.4 & 47.4 & 43.5 \\
$45 \mathrm{y}+$ & 13.1 & 12.3 & 13.8 & 12.0 \\
\hline TOTAL & 100.0 & 100.0 & 100.0 & 100.0 \\
\hline
\end{tabular}

$\mathrm{CHi}$ cuadrado $=137.1$ (G. L. $=9) \mathrm{p}<.001$

Diferencias estadisticamente significantes.

- Ibagué y Pasto difieren significativamente de Cali y P.A.M.I.

- (El promedio nacional de población 15 años es $40 \%$ )

Tabla No. 2. La distribución etárea de la población de Cali es similar a la del P.A.M.I. en Bogotá. Ibagué y Pasto tienen poblaciones más jóvenes (Ibagué 42.3, Pasto 44.5 de menores de 15 años respectivamente).

Pasto tiene la mayor población de menores de 1 año.
Tabla No. 3

INDICE DE MASCULINIDAD

\begin{tabular}{|lr|}
\hline Ciudad & I.M. (O/O) \\
\hline Cali & 96.1 \\
Ibagué & 90.8 \\
Bogotá (P.A.M.I.) & 97.1 \\
Pasto & 98.4 \\
CHi cuadrado $=11.46$ (G.L. $=3$ ) \\
\multicolumn{2}{|c|}{ p < .05 } \\
Diferencias estadísticamente \\
significantes
\end{tabular}

Tabla No. 3. Por cada 100 mujeres hay en Cali 96 hombres, P.A.M.I. 97 y Pasto 98. En Ibagué sólo hay 91 , cuál es estadísticamente significante.

Tabla No. 4

\section{PORCENTAJE DE MUJERES} EN EDAD

REPRODUCTIVA EN RELACION A LA POBLACION TOTAL

\begin{tabular}{|c|c|}
\hline Ciudad & $\begin{array}{c}0 / 0 \\
(15-44)\end{array}$ \\
\hline Cali & 25.0 \\
\hline Ibagué . & 24.6 \\
\hline Bogotá (P.A.M.I.) & 23.7 \\
\hline Pasto & 22.8 \\
\hline \multicolumn{2}{|c|}{$\begin{aligned} \mathrm{CHi} \text { cuadrado }= & 14.57 \quad(\mathrm{G} . \mathrm{L} .=3) \\
& p<.05\end{aligned}$} \\
\hline $\begin{array}{l}\text { Diferencias estadísticamente } \\
\text { significantes }\end{array}$ & \\
\hline
\end{tabular}

Cali difiere significativamente. Ibagué también.

$\overline{\mathrm{X}} \mathrm{Nal} . \quad$ : 22.4

$\overline{\mathrm{X}}$ Cali $\quad: 27.1$

Tabla No. 4. La proporción de mujeres en edad reproductiva en relación a la población total es mayor en Cali $(25 \%)$ y menor en Pasto $(22.8 \%)$. 
Tabla No. 5

PROPORCION DE EMBARAZOS (POR $10^{3}$ DE 15-44)

\begin{tabular}{|lc|}
\hline Ciudad & $\begin{array}{c}\text { O/o } \\
\text { Embarazo }\end{array}$ \\
\hline Cali & 93.3 \\
Ibagué & 152.3 \\
Bogotá (P.A.M.I.) & 109.5 \\
Pasto & 121.9 \\
CHi cuadrado = 98 (G.L. $=3)$ \\
\multicolumn{2}{c}{ p $<$.05 } \\
Diferencias estadísticamente \\
significantes.
\end{tabular}

Tabla No. 6

TASAS DE FECUNDIDAD GENERAL $\left(10^{3} \underline{0}\right)$ y NATALIDAD (10 ${ }^{3}$ HAB.)

\begin{tabular}{|l|c|c|}
\hline Ciudad & $\begin{array}{c}\text { Fecundidad } \\
\text { General } \\
\left(10^{3} \text { }\right)\end{array}$ & $\begin{array}{c}\text { Natalidad } \\
\left(10^{3} \mathrm{Hab} .\right)\end{array}$ \\
\hline Cali & 87.8 & 21.9 \\
\hline Ibagué & 139.4 & 34.3 \\
\hline $\begin{array}{l}\text { Bogotá } \\
\text { (P.A.M.I })\end{array}$ & 108.1 & 25.6 \\
\hline Pasto & 204.8 & 46.6 \\
\hline
\end{tabular}

$1.978-75 \%$ Valle

$\bar{X}$ Nacional Natalidad : 29.1

$\bar{X}$ Valle Natalidad : 16.8

1979

$\bar{X}$ Nacional fecundidad : 108.6

$\bar{X}$ Valle fecundidad : 75.

Natalidad

: $23 \% \quad 1979$

Fecundidad

: $79 \% \quad 1979$
Tabla No. 5. Hay mayor proporción de mujeres que se embarazan, por 1.000 mujeres en edad reproductiva en Ibagué (152.3) y en Pasto (121.9), desciende en el P.A.M.I. (109.5) y el menor porcentaje se encuentra en Cali, lo que nos sugiere una mayor utilización de mé. todos de control de natalidad en esta última.

Tabla No. 7

MORTALIAD GENERAL (TASAS $\times 10^{3}$ H.)

\begin{tabular}{|l|c|c|}
\hline Ciudad & $\begin{array}{c}\text { Tasa } \\
\text { Mortalidad } \\
\text { General }\end{array}$ & $\begin{array}{c}\text { Tasa } \\
\text { Ajustada } \\
\left({ }^{*}\right)\end{array}$ \\
\hline Cali & 4.8 & 4.8 \\
Ibagué & 4.0 & 4.1 \\
Bogotá (P.A.M.I.) & 6.8 & 6.6 \\
Pasto & 12.0 & 10.3 \\
\hline
\end{tabular}

(*) Ajuste por el Método Directo. Se utilizó como población standard la suma de las poblaciones.

$\begin{array}{llll}\text { - } & \text { Mortalidad en Valle } & : & 5 \\ \text { - } & \text { Mortalidad general Col. } & : & 8 \\ & \text { (Minsalud) } 1978\end{array}$

6.7

(Encuesta Nacional de Hogares). $\mathrm{VI} / 78$.

Tabla No. 7. La mortalidad general, por 1.000 habitantes, más alta se encuentra en Pasto (10.3), dobla la de Cali e Ibagué; estas dos últimas se encuentran por debajo del pro. medio nacional de 6.7. Similar a la del P.A.M.I.

Tabla No, 6. La mayor fecundidad se en. cuentra en Pasto (204.8) que dobla el prome. dio nacional y la menor en Cali (87.8). Igual comportamiento tiene la natalidad. 
Tabla No. 8

MORTALIDAD INFANTIL $\left(10^{3}\right.$ N. V.)

\begin{tabular}{|lc|}
\hline Ciudad & $\begin{array}{c}\text { Tasa } \\
\text { Mortalidad } \\
\text { Infantil }\end{array}$ \\
\hline Cali & 50.0 \\
Ibagué & 32.2 \\
Bogotá (P.A.M.I.) & 33.1 \\
Pasto & 105.3 \\
\hline
\end{tabular}

- 83 - 77 Minsalud - 1978.

- 69 Dane-Encuesta Nacional de hogares - VI/78

Tabla No. 8. La mortalidad infantil (menores de 1 año por 1.000 nacidos vivos) a excepción de Pasto, se encuentra en las ciudades restantes por debajo del promedio nacional, pero continúa siendo 2 veces la de Estados Unidos en Ibagué y P.A.M.I. y 3 veces en Cali. Pasto es el doble de la de Cali.

Tabla No. 9

PORCENTAJE DE NIUERTES EN MENORES DE UN AÑO CON RESPECTO AL TOTAL DE MUERTES

\begin{tabular}{|lc|}
\hline Ciudad & $\begin{array}{c}\text { O/o } \\
\text { Muertes }\end{array}$ \\
\hline Cali & 23.7 \\
Ibagué & 27.4 \\
Bogotá (P.A.M.I.) & 12.5 \\
Pasto & 41.4 \\
\hline
\end{tabular}

Tabla No. 9. A la mortalidad general de la población las muertes en niños menores de 1 año contribuyen grandemente con porcentajes que varía entre 12.5 en P.A.M.I. (Bogotá) y $41.4 \%$ en Pasto. Cali contribuye con el 23.7 e Ibagué con el 27.4 .
Tabla No. 10

DISTRIBUCION PORCENTUAL POR GRUPOS DE EDAD DE LAS GESTANTES - 1980

\begin{tabular}{|c|c|c|c|c|}
\hline $\begin{array}{l}\text { Ėdad } \\
\text { (años) }\end{array}$ & $\begin{array}{l}\text { Cali } \\
(0 \%)\end{array}$ & $\begin{array}{l}\text { Ibagué } \\
(0 / 0)\end{array}$ & $\begin{array}{c}\text { Bogotá } \\
\text { (P.A.M.I.) } \\
(0 \%)\end{array}$ & $\begin{array}{l}\text { Pasto } \\
(0 / 0)\end{array}$ \\
\hline $15-9$ & 22.9 & 13.0 & 6.5 & 9.7 \\
\hline $20-24$ & 35.0 & 33.2 & 30.7 & 31.7 \\
\hline $25-29$ & 20.9 & 24.8 & 26.1 & 23.9 \\
\hline $30-34$ & 10.8 & 15.3 & 21.6 & 18.2 \\
\hline $35-39$ & 7.6 & 8.8 & 13.1 & 10.0 \\
\hline $40-44$ & 2.3 & 3.7 & 1.9 & 5.0 \\
\hline TOTAL & 100.0 & 100.0 & 100.0 & 100.0 \\
\hline \multicolumn{3}{|c|}{$(\mathrm{N}=882)(\mathrm{N}=431)$} & $(N=153)$ & $(N=259)$ \\
\hline EAR: & 33.3 & 26.7 & 21.5 & 26.2 \\
\hline
\end{tabular}

Diferencias estadisticamente significantes:

Tabla No. 10. En Cali se encuentra el grupo de madres más jóvenes que en las demás ciu. dades, en donde se observan embarazos más tardíos, en mujeres mayores de 35 años.

Se encuentra la mayor proporción de em. barazos de alto riesgo por edad (menores de 20 años más las de 35 años y más) en Cali $(33.3 \%)$, en lbagué corresponde al 26.7 , en Pasto al 26.2 y la menor se encuentra en el P.A.M.I.

Tabla No. 11

\section{PROMEDIO DE PARTOS}

\begin{tabular}{|lc|}
\hline \multicolumn{1}{|c|}{ Ciudad } & $\begin{array}{c}\overline{\mathrm{X}} \\
\text { Partos }\end{array}$ \\
\hline Cali & 2.6 \\
Ibagué & 2.9 \\
Bogotá (P.A.M.I.) & 2.5 \\
Pasto & 3.0 \\
\hline - Cali y Pasto + = 2.18 (G.L. $=1138$ ) \\
p < .05
\end{tabular}

Diferencias estadísticamente significantes. 
Tabla No. 11. El promedio de paridad varió de 2.5 en el P.A.M I. a 3 en Pasto, estas dife. rencias significantes.

En Cali - Ibagué, P.A.M.I. más del 90\% de las gestantes asistieron por lo menos una vez a control prenatal.

En Pasto el $37 \%$ de las gestantes no tuvieron ningún control.

Tabla No. 12. En Cali, Ibagué y Pasto sólo la mitad de las gestantes que asisten a control prenatal lo hacen en el 1er. trimestre Sólo en el P.A.M.I. asisten más precozmente. Las consultas se incrementan en el 20. $\vee$ 3o. trimestre.

El P.A.M.I. se destacá como el de mayor cobertura en todos los trimestres.
Tabla No. 12

$\%$ GESTANTES QUE

FUERON A CONTROL PRENATAL. SEGUN TRIMESTRE

DE GESTACION

\begin{tabular}{|l|c|c|c|}
\hline \multirow{2}{*}{\multicolumn{1}{|c|}{ Ciudad }} & \multicolumn{3}{c|}{$\begin{array}{c}\text { \% Control } \\
\text { Según Trimestre }\end{array}$} \\
\cline { 2 - 4 } & I & II & III \\
\hline Cali & 56.1 & 76.6 & 81.8 \\
Ibagué & 49.2 & 66.1 & 63.1 \\
Bogotá (P.A.M.I.) & 75.3 & 92.0 & 97.0 \\
Pasto & 54.8 & 40.9 & 49.4 \\
\hline
\end{tabular}

Tabla No. 13

DISTRIBUCION PORCENTUAL SEGUN LUGAR DEL CONTROL PRENATAL

\begin{tabular}{|l|c|c|c|c|}
\hline $\begin{array}{c}\text { Lugar del } \\
\text { Control }\end{array}$ & $\begin{array}{c}\text { Cali } \\
(\% / \mathrm{o})\end{array}$ & $\begin{array}{c}\text { Ibagué } \\
(\mathrm{O} / \mathrm{o})\end{array}$ & $\begin{array}{r}\text { Bogotá (P.A.M.I.) } \\
(\mathrm{O} / \mathrm{o})\end{array}$ & $\begin{array}{r}\text { Pasto } \\
(\mathrm{O} / \mathrm{O})\end{array}$ \\
\hline Centro de salud & 53.4 & 40.8 & 50.7 & 52.1 \\
I. S. S. & 24.5 & 19.7 & 27.3 & 12.3 \\
Hospital & 10.6 & 19.7 & 7.3 & 16.6 \\
Médico & 4.9 & 9.9 & 14.0 & 8.6 \\
Particular & 3.4 & 1.9 & .7 & 5.5 \\
Casa & 3.2 & 8.0 & - & 4.9 \\
Otros & 100.0 & 100.0 & 100.0 & 100.0 \\
\hline \multicolumn{1}{|c|}{ TOTAL } & $(\mathrm{N}=714)$ & $(\mathrm{N}=375)$ & $(\mathrm{N}=153)$ & $(\mathrm{N}=163)$ \\
\hline
\end{tabular}

$\mathrm{CHi}$ cuadrado $=174.4$ (G.L. $=9$ ) $p<0.05$

Diferencias estadísticamente significantes

Tabla No. 13. El control prenatal es institucional (Centro de Salud, ISS, hospital) en más del $80 \%$ de las gestantes, siendo el mayor porcentaje en Cali.
Llama la atención cómo en el P.A.M.I. se encuentra la mayor proporción de controles por médico particular; el menor se encuentra en Cali. 
Tabla No. 14

PERSONA ENCARGADA DEL CONTROL PRENATAL

\begin{tabular}{|c|c|c|c|c|}
\hline $\begin{array}{c}\text { Encargada. del } \\
\text { Control }\end{array}$ & $\begin{array}{l}\text { Cali } \\
(\% / 0)\end{array}$ & $\begin{array}{l}\text { Ibagué } \\
(0 \% \text { o) }\end{array}$ & $\begin{array}{c}\text { Bogotá (P.A.M.I.) } \\
(\%)\end{array}$ & $\begin{array}{l}\text { Pasto } \\
(0 / 0)\end{array}$ \\
\hline Médico & 51.3 & 83.0 & 48.6 & 77.3 \\
\hline Auxiliar & 24.7 & 1.6 & - & 2.5 \\
\hline Enfermera & 8.5 & 3.2 & - & 3.7 \\
\hline Partera & 2.9 & 1.9 & .7 & 6.1 \\
\hline $\begin{array}{l}\text { Médico o Enfermera } \\
\text { Auxiliar }\end{array}$ & 11.1 & 10.0 & 50.7 & 9.8 \\
\hline $\begin{array}{l}\text { Enfermera y } \\
\text { Auxiliar }\end{array}$ & 1.5 & .3 & - & .6 \\
\hline TOTAL & 100.0 & 100.0 & 100.0 & 100.0 \\
\hline
\end{tabular}

$-\mathrm{CHi}$ cuadrado $=331.9$ (G.L. $=9) \mathrm{p}<0.05$ Diferencias estadísticamente significantes.

Tabla No. 14. En los $2 / 3$ de las gestantes en Ibagué y Pasto el control es realizado exclusivamente por médico.

En Cali y P.A.M.I. es la mitad.
Hay una marcada delegación del control en la enfermera y la auxiliar en Cali.

El mayor control por partera se encuentra en Pasto.

Tabla No. 15

FORMA DE TERMINACION DEL EMBARAZO

\begin{tabular}{|c|c|c|c|c|}
\hline $\begin{array}{l}\text { Forma de Terminación } \\
\text { dél Parto }\end{array}$ & $\begin{array}{l}\text { Cali } \\
(0 / 0)\end{array}$ & $\begin{array}{l}\text { Ibagué } \\
(0 \%)\end{array}$ & $\begin{array}{c}\text { Bogotá (P.A.M.I.) } \\
(\mathrm{O} \%)\end{array}$ & $\begin{array}{l}\text { Pasto } \\
(0 / 0)\end{array}$ \\
\hline Normal & 88.0 & 87.0 & 82.4 & 91.5 \\
\hline Cesárea & 7.5 & 6.7 & 13.7 & 4.6 \\
\hline Parto Prematuro & 2.6 & 1.4 & 2.0 & 1.5 \\
\hline Aborto primer trimestre & .6 & 3.7 & 1.3 & 1.5 \\
\hline Aborto Segundo trimestre & 1.0 & 1.2 & .7 & .8 \\
\hline Cesárea, parto prematuro & .2 & - & - & - \\
\hline Sin dato & .1 & - & - & - \\
\hline TOTAL & 100.0 & 100.0 & 100.0 & 100.0 \\
\hline - \% Abortos: & 1.6 & 4.9 & 2.0 & 2.3 \\
\hline
\end{tabular}

$-\mathrm{CHi}^{2}=26.9$ (G.L. $\left.=9\right) \mathrm{p}<0.05$

Diferencias estadísticamente significantes.

Tabla No. 15. Las gestaciones terminaron en parto normal en Ibagué $(87 \%)$ Cali (88) Pasto (91.5) y P.A.M.I. (82.4).

El porcentaje de cesáreas varió entre 4.6 en Pasto, 6.7 en Ibagué, Cali 7.5 y el mayor
13.7 en el P.A.M.I. El parto prematuro varió entre 1.4 Ibagué y 2.6 en Cali. La proporción de abortos en Ibagué es el doble de las ciuda des restantes. 
Tabla No. 16

LUGAR DE ATENCION DEL PARTO

\begin{tabular}{|l|c|c|c|c|}
\hline Lugar de Atención & $\begin{array}{c}\text { Cali } \\
(\% / 0)\end{array}$ & $\begin{array}{c}\text { Ibagué } \\
(\% / 0)\end{array}$ & $\begin{array}{c}\text { Bogotá (P.A.M.I.) } \\
(\mathrm{O} / \mathrm{O})\end{array}$ & $\begin{array}{c}\text { Pasto } \\
(\mathrm{O} / \mathrm{o})\end{array}$ \\
\hline Hospital & 28.1 & 34.5 & 37.4 & 47.2 \\
I. S. S. & 23.5 & 9.7 & 32.7 & 15.8 \\
Casa & 20.6 & 40.7 & 7.5 & 34.0 \\
Centro de Salud & 23.7 & 4.4 & 8.8 & .4 \\
Clínica particular & 3.4 & 8.6 & 13.6 & 1.2 \\
Otros & .7 & 2.1 & - & 1.5 \\
\hline \multicolumn{1}{|c|}{ TOTAL } & 100.0 & 100.0 & 100.0 & 100.0 \\
\hline
\end{tabular}

$\mathrm{CHi}$ cuadrado $=226.5($ G.L. $\times 12) \mathrm{p}<0.05$ Diferencias estadísticamente significantes

Tabla No. 16. El parto es atendido en la mayoría de los casos institucionalmente, sin embargo en Ibagué el $40.7 \%$ son en casa, en
Pasto el $34 \%$, en Cali el 20.6 y en el P.A.M.I. $7.5 \%$.

Tabla No. 17

PERSONA ENCARGADA DE LA ATENCION DEL PARTO

\begin{tabular}{|l|r|c|c|c|}
\hline $\begin{array}{c}\text { Persona que atendió } \\
\text { el Parto }\end{array}$ & $\begin{array}{c}\text { Cali } \\
(\% / 0)\end{array}$ & $\begin{array}{c}\text { Ibagué } \\
(\% / 0)\end{array}$ & $\begin{array}{c}\text { Bogotá (P.A.M.I.) } \\
(\%)\end{array}$ & $\begin{array}{c}\text { Pasto } \\
(\% / 0)\end{array}$ \\
\hline Médico & 53.4 & 49.6 & 90.9 & 60.2 \\
Partera & 17.0 & 31.1 & 5.7 & 26.6 \\
Auxiliar & 15.8 & 6.4 & 1.7 & 2.3 \\
Enfermera & 11.5 & 10.5 & 1.7 & 3.9 \\
M. D., Enfermera & .4 & 1.7 & - & - \\
M.D., Auxiliar & .2 & .5 & - & - \\
Otros & 1.7 & .2 & - & 7.0 \\
\hline \multicolumn{1}{|c|}{ TOTAL } & 100.0 & 100.0 & 100.0 & 100.0 \\
\hline
\end{tabular}

Tabla No. 17. El parto en la mitad de los casos en Cali, Ibagué y Pasto es atendido por médico. En el P.A.M.I, en el $91 \%$.

La partera tradicional es un personaje muy importante en Ibagué (31.1) Pasto (26.6) y aún en Cali $(17 \%)$. En Cali es muy importante la auxiliar $y$ enfermera (27.3) y en Ibagué (16.9). 
Tabla No. 18

DISTRIBUCION DE LAS GESTANTES SEGIJN TIPO DE PARTO

\begin{tabular}{|l|r|c|c|c|}
\hline $\begin{array}{c}\text { Tipo de } \\
\text { Parto }\end{array}$ & Cali & Ibagué & Bogotá (P.A.M.I.) & Pasto \\
\hline Unico & 98.4 & 99.0 & 98.6 & 97.8 \\
Múltiple & 1.6 & 1.0 & 1.4 & 2.2 \\
\hline TOTAL & 100.0 & 100.0 & 100.0 & 100.0 \\
\hline
\end{tabular}

Tabla No. 18. Características del parto.

La gran mayoría de los partos fueron únicos,

a término. El parto múltiple varió, entre el
$1 \%$ en Ibagué, P.A.M.I. 1.4, Cali 1.6 y Pasto el $2.2 \%$.

Tabla No. 19

EDAD DE GESTACION DEL NIÑO

\begin{tabular}{|c|c|c|c|c|}
\hline $\begin{array}{c}\text { Edad } \\
\text { Gestación } \\
\text { niño }\end{array}$ & $\begin{array}{c}\text { Cali } \\
(\mathrm{O} / \mathrm{O})\end{array}$ & $\begin{array}{c}\text { Ibagué } \\
(\mathrm{O} / \mathrm{O})\end{array}$ & $\begin{array}{r}\text { Bogotá (P.A.M.I.) } \\
(\mathrm{O} / \mathrm{O})\end{array}$ & $\begin{array}{r}\text { Pasto } \\
(\mathrm{O} / \mathrm{O})\end{array}$ \\
\hline A término & 92.8 & 95.0 & 99.3 & 94.8 \\
De 7-8 meses & 7.2 & 5.0 & .7 & 5.2 \\
\hline TOTAL & 100.0 & 100.0 & 100.0 & 100.0 \\
\hline
\end{tabular}

Tabla No. 19. El parto prematuro es el Pasto y muy baja 0.7 en P.A.M.I. $7.2 \%$ en Cali, el $5 \%$ en Ibagué y 5.2 en

Tabla No. 20

FORMA DE PRESENTACION DEL NIÑO

\begin{tabular}{|l|c|c|c|c|}
\hline $\begin{array}{c}\text { Presentación } \\
\text { del niño }\end{array}$ & $\begin{array}{c}\text { Cali } \\
(\mathrm{O} / \mathrm{o})\end{array}$ & $\begin{array}{c}\text { Ibagué } \\
(\mathrm{O} / \mathrm{O})\end{array}$ & $\begin{array}{c}\text { Bogotá (P.A.M.I.) } \\
(\mathrm{O} / \mathrm{O})\end{array}$ & $\begin{array}{r}\text { Pasto } \\
(\mathrm{O} / \mathrm{o})\end{array}$ \\
\hline De cabeza & 91.5 & 95.9 & 93.2 & 90.5 \\
Sentado & 3.3 & 3.6 & 6.8 & 5.2 \\
Otra & 5.2 & .5 & 6.8 & 4.3 \\
\hline TOTAL & 100.0 & 100.0 & 100.0 & 100.0 \\
\hline
\end{tabular}

CHi cuadrado $=7.7(\mathrm{GL}=3) \quad \mathrm{p}<0.05$

Diferencias significantes

Tabla No. 2C. La presentación cefálica en más del $90 \%$ en todas las ciudades. En Cali
$(8.5 \%)$ y Pasto $(9.5 \%)$, son partos con otras presentaciones. 


\section{DISTRIBUCION PORCENTUAL SEGUN TIPO DE COMPLICACION DEL NIÑO}

\begin{tabular}{|l|c|c|c|c|}
\hline $\begin{array}{c}\text { Complicaciones } \\
\text { del niño }\end{array}$ & $\begin{array}{c}\text { Cali } \\
(\mathrm{O} / \mathrm{O})\end{array}$ & $\begin{array}{c}\text { Ibagué } \\
(\mathrm{O} / \mathrm{O})\end{array}$ & $\begin{array}{c}\text { Bogotá (P.A.M.I.) } \\
(\mathrm{O} / \mathrm{O})\end{array}$ & $\begin{array}{r}\text { Pasto } \\
(\mathrm{O} / \mathrm{o})\end{array}$ \\
\hline Ninguna & 86.0 & 78.4 & 99.3 & 84.2 \\
Demora en respirar & 4.7 & 5.0 & - & 1.4 \\
Morado & 4.7 & 6.9 & - & 4.6 \\
Fue hospitalizado & 2.8 & 1.0 & .7 & 2.3 \\
$\begin{array}{l}\text { Demora en respirar } \\
\text { o morado o fue } \\
\text { hospitalizado }\end{array}$ & 1.8 & 8.7 & - & \\
\hline \multicolumn{1}{|c|}{ TOTAL } & 100.0 & 100.0 & 100.0 & 100.0 \\
\hline
\end{tabular}

CHi cuadrado $=39.6$ (G.L. $=3$ ) $p<0.05$ Diferencias estadisticamente significantes.

Tabla No. 21. Los niños se complican al nacer más frecuentemente $(21.6 \%)$ en Ibagué; en Pasto el $15.8 \%$, en Cali el $14 \%$ y sólo el 0.7 en el P.A.M.I.

\section{DISTRIBUCION PORCENTUAL SEGUN TIPO DE COMPLICACION MATERNA DURANTE EL PARTO O ABORTO}

\begin{tabular}{|c|c|c|c|c|}
\hline $\begin{array}{l}\text { Complicaciones } \\
\text { maternas }\end{array}$ & $\begin{array}{l}\text { Cali } \\
(0 / 0)\end{array}$ & $\begin{array}{c}\text { Ibagué } \\
(0 / 0)\end{array}$ & $\begin{array}{c}\text { Bogotá (P.A.M.I.) } \\
(0 / 0)\end{array}$ & $\begin{array}{l}\text { Pasto } \\
(0 / 0)\end{array}$ \\
\hline Ninguna & 33.2 & 80.0 & 62.8 & 76.4 \\
\hline $\begin{array}{l}\text { Hemorragia durante } \\
\text { Parto o Aborto }\end{array}$ & 4.5 & 6.7 & - & 3.5 \\
\hline $\begin{array}{l}\text { Hemorragia Post } \\
\text { Parto o Aborto }\end{array}$ & 3.3 & 4.4 & 2.7 & 12.4 \\
\hline Desgarros & 2.7 & 4.4 & 21.6 & 3.9 \\
\hline Fiebre hasta de 8 dias & 3.6 & 3.0 & 12.8 & 2.7 \\
\hline Otras & 2.7 & 1.5 & - & 1.1 \\
\hline TOTAL & 100.0 & 100.0 & 100.0 & 100.0 \\
\hline
\end{tabular}

Mortalidad Materna $=9.6 \%$

(Edad Representativa)

CHi cuadrado $=7.7$ (G.L. $=3$ ) p $<05$

Diferencias no significantes.

Tabla No. 22. La madre durante el parto o aborto no se complica en el 83.2 en Cali, 80 en Ibagué, Pasto $76.4 \%$.

En el P.A.M.I. se encuentra la mayor proporción de complicaciones tipo desgarres (21.6) y fiebre hasta de 8 días (12.8).

La hemorragia post-parto 0 aborto es más frecuente en Pasto (12.4\%.

La hemorragia durante el parto o aborto es la complicación más frecuente en Cali $(4.5 \%)$ en Ibagué (6.7).

\section{CONSULTA (\%) DESPUES DEL PARTO O ABORTO}

\begin{tabular}{|c|c|c|c|c|}
\hline Consulta & $\begin{array}{c}\text { Cali } \\
(\mathrm{O} / 0)\end{array}$ & $\begin{array}{c}\text { Ibagué } \\
(\mathrm{O} / \mathrm{O})\end{array}$ & $\begin{array}{c}\text { Bogotá (P.A.M.I.) } \\
(\mathrm{O} / \mathrm{o})\end{array}$ & $\begin{array}{c}\text { Pasto } \\
(\mathrm{O} / \mathrm{0})\end{array}$ \\
\hline $\mathrm{Si}$ & 55.5 & 32.3 & 47.9 & 29.0 \\
$\mathrm{NC}$ & 44.5 & 67.7 & 52.1 & 71.0 \\
\hline TOTAL & 100.0 & 100.0 & 100.0 & 100.0 \\
\hline
\end{tabular}

CHi cuadrado $=95.2(\mathrm{G} . \mathrm{L} .=3) p .05$

Diferencias estadisticamente significantes

Tabla No. 23. En Cali y P.A.M.I. sólo la mitad de las gestantes asisten a consulta post. parto ó aborto. En lbagué y Pasto las $2 / 3$ no consultaron.

\section{LUGAR DE CONSULTA $(\%)$ POST-PARTO O ABORTO}

\begin{tabular}{|l|c|c|c|c|}
\hline $\begin{array}{c}\text { Lugar de } \\
\text { Consutta }\end{array}$ & $\begin{array}{c}\text { Cali } \\
(\mathrm{O} / 0)\end{array}$ & $\begin{array}{c}\text { (bagué } \\
(\mathrm{O} \%)\end{array}$ & $\begin{array}{c}\text { Bogotá (P.A.M.I.) } \\
(\mathrm{O} / 0)\end{array}$ & $\begin{array}{c}\text { Pasto } \\
(\mathrm{O} \%)\end{array}$ \\
\hline Centro de salud & 46.7 & 45.8 & 47.1 & \\
I. S. S. & 21.1 & 14.8 & 17.6 & \\
Hospital & 12.4 & 20.4 & 1.5 & \\
Casa & 6.8 & 4.2 & 20.6 & \\
Clínica particular & 6.2 & 14.8 & 13.2 & \\
Otros & 6.8 & - & - & \\
\hline \multicolumn{1}{|c|}{ TOTAL } & 100.0 & 100.0 & 100.0 & \\
\hline
\end{tabular}

Tabla No. 24. Las que consultan lo hacen a instituciones oficiales.

\section{CONTROL DEL NIÑO SANO (\%)}

\begin{tabular}{|c|c|c|c|c|}
\hline $\begin{array}{c}\text { Control } \\
\text { Niño } \\
\text { Sano }\end{array}$ & $\begin{array}{c}\text { Cali } \\
(\%)\end{array}$ & $\begin{array}{c}\text { Ibagué } \\
(\%)\end{array}$ & $\begin{array}{c}\text { Bogotá (P.A.M.I.) } \\
(\% \%)\end{array}$ & $\begin{array}{c}\text { Pasto } \\
(\% / 0)\end{array}$ \\
\hline $\mathrm{Si}$ & 46.2 & 37.1 & 91.7 & 44.5 \\
No & 53.8 & 62.9 & 8.3 & 55.5 \\
\hline TOTAL & 100.0 & 100.0 & 100.0 & 100.0 \\
\hline
\end{tabular}

CHi cuadrado $=138.9$ (G.L. $=3) p<0.05$

Diferencias Estadísticamente significantes.

Tabla No. 25. Un poco más de la mitad de los niños no son llevados al control del niño sano en Cali, Ibagué, Pasto. Sólo en el P.A.M.I. es el $91.7 \%$. 
Tabla No. 26

TASAS DE MORTINATALIDAD NEONATAL Y PERINATAL $\left(10^{3} \mathrm{~N}\right.$. V.)

\begin{tabular}{|l|r|r|r|}
\hline $\begin{array}{c}\text { Tasas de } \\
\text { Mortalidad }\end{array}$ & $\begin{array}{c}\text { Cali } \\
(\%)\end{array}$ & $\begin{array}{r}\text { (bagué } \\
(\% \%)\end{array}$ & $\begin{array}{r}\text { Pasto } \\
(\%)\end{array}$ \\
\hline Al nacimiento & 28.3 & 7.0 & 13.2 \\
8 días & 8.1 & 9.9 & 26.3 \\
$8-28$ días & 5.8 & 9.8 & 4.3 \\
Neonatal & 13.9 & 19.7 & 30.6 \\
Perinatal I & 36.4 & 16.9 & 39.5 \\
\hline
\end{tabular}

\begin{tabular}{|l|c|c|}
\hline \multicolumn{2}{|c|}{$\begin{array}{c}\text { Mortalidad } \\
\text { Perinatal }\end{array}$} & $\begin{array}{c}\text { Mortalidad } \\
\text { Neonatal }\end{array}$ \\
\hline Méjico & 29.1 & 19.5 \\
Panamá & 27.4 & 14.5 \\
Perú & 39.9 & 18.1 \\
Repúbl. Dom. & 26.3 & 18.9 \\
Venezuela & & 17.4 \\
Cuba & 23.7 & 14.5 \\
\hline
\end{tabular}

Tabla No. 26. Las tasas de mortalidad perinatal por primera vez se obtienen para Cali, Ibagué y Pasto. No hay datos de esta morta* lidad para el país y son más altas que las repor. tadas por México, Panamá, República Dominicana y Cuba.

Son similares para Cali (36.4) y para Pasto (39.5). La más baja se encuentra en Ibagué. En Cali se observa mayor mortinatalidad y en Pasto e Ibagué mayor la mortalidad neonatal.

En Cali la mortalidad neonatal es similar aunque no comparable a la de Estados Unidos.

Debe profundizarse en el estudio de estas diferencias porque ha sido clara la mayor $\mathrm{co}^{\circ}$ bertura por el sistema de salud estatal para el control prenatal y parto institucional en Cali que no correlaciona con la menor mortalidad en Ibagué y mortalidad en Pasto. Se hacen necesario estudio sobre la calidad de atención.

\section{CAPITULO IV}

\section{Conclusiones y recomendaciones}

1. Obtenido el diagnóstico de base es conveniente profundizar en estudios que hagan claridad sobre las diferencias encontradas en los indicadores de salud materno-infantil, entre las ciudades y las regionales de salud investigadas.

2. Es conveniente introducir como norma la instalación de un sistema de vigilancia epidemiológica sobre la población materno-infantil, que permita información confiable y oportuna sobre los distintos indicadores de salud.

3. Se debe propiciar la vinculación de las sociedades científicas y el sector estatal de salud, que permita su participación y asesoría en la normatización de la atención de la madre y el niño, desde el nivel preventivo hasta el asistencial.

4. Utilizar los indicadores de salud por regionales, teniendo en cuenta que los promedios nacionales y departamentales no reflejan fielmente situaciones particulares de salud y enfermedad.

5. Deben continuarse los estudios de la salud de la madre y el niño que permitan un mejor conocimiento sobre lo que sucede a nivel de su atención en los programas estatales, para tomar medidas eficientes y oportunas.

6. Dado el desarrollo diferente de los servicios de atención médica en las secciones político-administrativas del país, es necesario un diagnóstico específico inicial para cada una de ellas, así como investigaciones regionales posteriores. 


\section{CAPITULO V}

\section{ANEXO 1 \\ Muestra de Cali.}

Marco Muestral: Con base en la información suministrada por Planeación Municipal se tomaron todos los barrios clasificados en el nivel socio-económico bajo, distribuidos según tamaño de la población.

El total de barrios clasificados en el nivel socio-económico bajo fue de 67 , con una población total estimada de 447.984 habitantes $(39.7 \%$ de la población de Cali).

Muestra: Con base en el tamaño de la población de cada barrio se formaron 5 estratos:

\begin{tabular}{|cr|}
\hline Estrato 1 (barrios menores $\triangle 2.000$ habitantes) $=13$ \\
Estrato 2 (barrios entre 2.000-4.999 habitantes) $=20$ \\
Estrato 3 (barrios entre $5.000-9.999$ habitantes) $=16$ \\
Estrato 4 (barrios entre 10.000-20.000 habitantes) $=16$ \\
Estrato 5 (barrios mayores $\triangle 20.000$ habitantes) $=2$ \\
\hline TOTAL & 67 \\
\hline
\end{tabular}

Cada barrio se consideró como un conglomerado.

Se tomó una muestra aleatoria proporcional al tamaño de cada estrato de 10 barrios, que cubren una población de 47.953 habitantes (equivalen aproximadamente a 7.052 viviendas). Esta muestra es igual al $10.7 \%$ de la población de Cali con nivel socio-económico bajo.

En cada barrio se entrevistaban todas las familias que viviesen en la casa (promedio de familias por vivienda: 1.3).

Los barrios seleccionados fueron: $\mathrm{Na}-$ cional, El Piloto, San Luis, San Marino, León XIII, Jorge Zawadsky, Santo Do- mingo, Lourdes, La Playa y Lleras Camargo.

Con un margen de error del $1.2 \%$, un límite de confianza del $95 \%$ y una probabilidad máxima de selección del $50 \%$, se requiere una muestra de 6.669 viviendas, muestra que se considera representativa de la población. Nuestra muestra fue de 7.052 viviendas.

\section{ESTUDIO MATERNO INFANTIL VALLE DEL CAUCA DISEÑO DE LA MUESTRA}

I. Definición del Marco Muestral. EI estudio se dirigió a analizar el problema materno infantil en la población con nivel socio-económico bajo, tanto en el área urbana como en el área rural del Valle del Cauca.

II Etapas en el Diseño de la Muestra. En la primera etapa del diseño se conformaron nueve grandes estratos naturales, que corresponden a las regionales en que está dividido el Valle del Cauca: Cali, Palmira, Buga, Tulúa, Cartago, Roldanillo, Zarzal, Sevilla y Buenaventura.

En cada ciudad se definieron las unidades primarias de muestreo tanto en el perímetro urbano como en el rural, utilizando el método de selección controlada.

Como cada regional está compuesta de niveles primarios, también se definieron en cada uno de ellos las unidades primarias, tanto en la parte urbana como en la parte rural.

En la segunda etapa, las unidades primarias seleccionadas se subdividieron en segmentos conformados por viviendas, para tomar luego una muestra aleatoria simple. 


\section{Muestra}

La muestra seleccionada corresponde al $8 \%$ del total de viviendas en el nivel socio-económico bajo en el Valle del Cauca.

\section{Fuentes de información}

4.1. Cali: Oficina de Planeación Municipal (clasificación de los barrios según nivel socio-económico y mapas); Departamento Nacional de Estadística (Impreso de Mayo/79 sobre Perfil Estadístico del Valle del Cauca) y documentos socio-demográficos del Servicio Seccional de Salud.

4.2. Palmira: Oficina de Planeación de las Empresas Municipales (mapas y estudio sobre nivel socio-económico).
4.3. Buga: Oficina de Planeación de la Alcaldía (mapas y estudio sobre nivel socio-económico).

4.4. Tulúa: Oficina de Planeación de la Alcaldía.

4.5. Cartago: Oficina de Planeación ción de la Alcaldía.

4.6. Buenaventura: Oficina de la C.V.C. para el Programa Regional de Buenaventura en Cali y Oficina de Planeación. valle.

4.7. Roldanillo: Oficina de Acuavalle.

4.8. Ginebra: Oficina de Catastro.

\section{MUESTRA SELECCIONADA EN EL VALLE}

\begin{tabular}{|lrrrrrr|}
\hline & $\begin{array}{c}\text { Realizado } \\
\text { Urbano }\end{array}$ & $\begin{array}{l}\text { Programado } \\
\text { Urbano }\end{array}$ & $\begin{array}{l}\text { Realizado } \\
\text { Rural }\end{array}$ & $\begin{array}{l}\text { Programado } \\
\text { Rural }\end{array}$ & $\begin{array}{l}\text { Realizado } \\
\text { Total }\end{array}$ & $\begin{array}{l}\text { Programado } \\
\text { Total }\end{array}$ \\
\hline Ginebra & 96 & 150 & 48 & 47 & 114 & 197 \\
Palmira & 770 & 800 & 239 & 367 & 1.009 & 1.167 \\
Restrepo & 69 & 67 & 78 & 39 & 147 & 106 \\
Buga & 356 & 370 & 102 & 65 & 458 & 435 \\
Andalucía & 147 & 150 & 100 & 47 & 247 & 197 \\
Tuluz & 268 & 529 & 299 & 204 & 567 & 733 \\
Ulloa & 51 & 43 & 39 & 40 & 90 & 83 \\
Cartago & 1.204 & 668 & 75 & 72 & 1.279 & 740 \\
Caicedonia & 282 & 378 & 54 & 81 & 336 & 459 \\
Sevilla & 1.573 & 991 & 178 & 178 & 1.751 & 1.125 \\
Bol ívar & 84 & 76 & 64 & 132 & 148 & 208 \\
Roldanillo & 152 & 181 & 131 & 147 & 283 & 328 \\
Versalles & 71 & 71 & 155 & 72 & 226 & 143 \\
Zarzal & 504 & 504 & 285 & 121 & 789 & 625 \\
La Cumbre & 77 & 113 & 95 & 67 & 172 & 180 \\
Cali & 5.841 & 6.482 & 107 & 160 & 5.948 & 6.642 \\
Buenaventura & 624 & 900 & & & 624 & 900 \\
\hline TOTALES & 12.169 & 12.473 & 2.049 & 1.795 & 14.218 & 14.268 \\
\hline
\end{tabular}




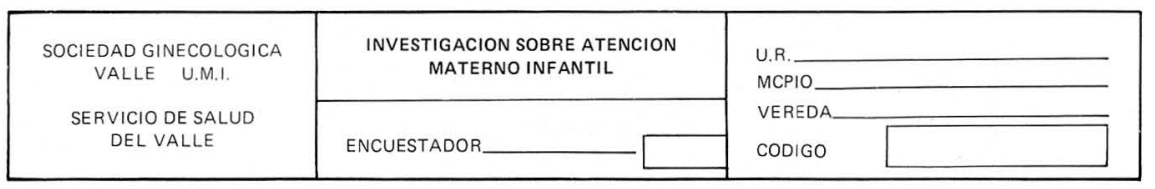

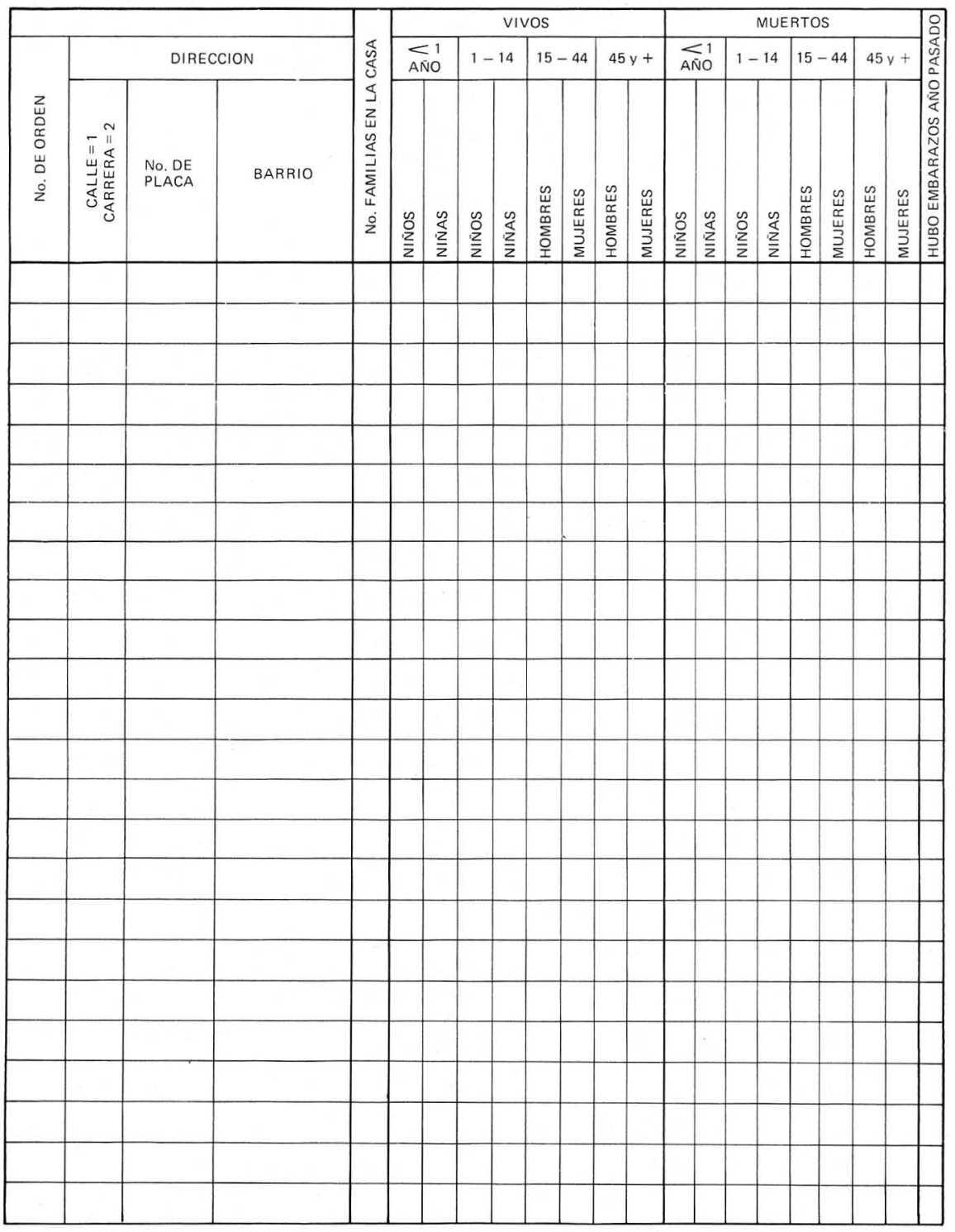


SOCIEDAD VALLECAUCANA DE OBSTETRICIAY GINECOLOGIA

Sefvicio de salud del valle

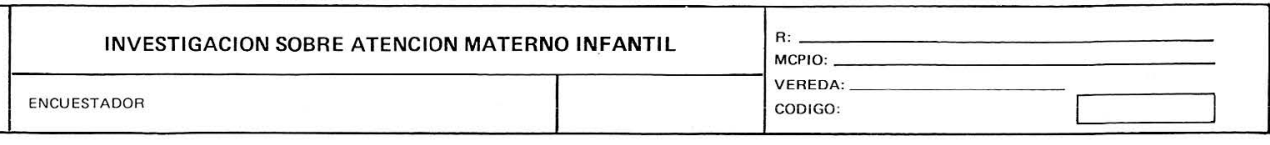

\begin{tabular}{|c|c|c|c|c|c|c|c|c|c|c|c|c|c|c|c|c|c|c|c|c|c|c|c|c|c|c|c|c|c|c|c|}
\hline$\frac{\pi}{8}$ & & $\begin{array}{l}\text { DATC } \\
\text { ZADA }\end{array}$ & $\begin{array}{l}S S D E \\
A S D E \\
A S A S A\end{array}$ & $\begin{array}{l}\text { EMB } \\
\text { LAN } \\
\text { DON }\end{array}$ & $\begin{array}{l}\text { ARA. } \\
0\end{array}$ & & $\begin{array}{l}\text { DA } \\
\text { SOB } \\
\text { ONT } \\
\text { REEN }\end{array}$ & & & $\begin{array}{l}\text { DA } \\
\text { ATE } \\
\text { RTO }\end{array}$ & $\begin{array}{l}\text { ATOS } \\
\text { ENCIO }\end{array}$ & $\begin{array}{l}\text { OBRE } \\
\text { N DEL } \\
\text { SRTO }\end{array}$ & iv & & & $\begin{array}{l}\text { ATOS } \\
\text { REE } \\
\text { BTO }\end{array}$ & & $\begin{array}{l}\text { VI. CO } \\
\text { COONES } \\
\text { NAS } \\
\text { ART }\end{array}$ & $\begin{array}{l}\text { MPLIC } \\
\text { MAT } \\
\text { OABR } \\
\text { ABB }\end{array}$ & & $\begin{array}{r}\text { VII.DA } \\
\text { SOB } \\
\text { ELLNI }\end{array}$ & & & VIII. EL NINO MURIO & & & & $\begin{array}{l}\text { EL NII } \\
\text { ACTU }\end{array}$ & $\begin{array}{l}\text { Vo VI } \\
\text { ALME }\end{array}$ & & \\
\hline 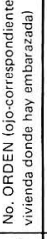 & 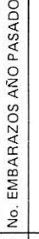 & 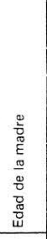 & 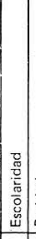 & 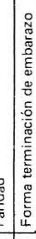 & 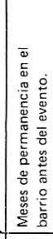 & 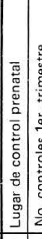 & 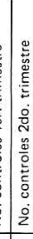 & 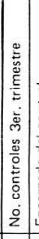 & 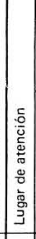 & 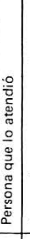 & 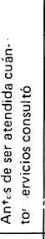 & 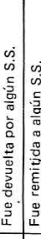 & 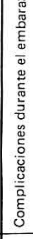 & 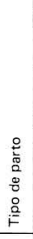 & 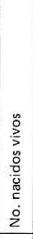 & 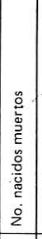 & & \begin{tabular}{l|l} 
\\
$\vdots$ \\
0
\end{tabular} & 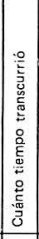 & 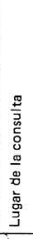 & 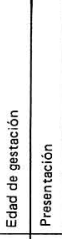 & 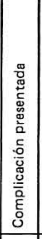 & 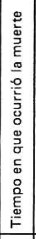 & $\begin{array}{l}\text { CAUSA DE LA MUERTE DEL } \\
\text { NIÑO }\end{array}$ & CODIGO & & 总 & 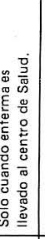 & 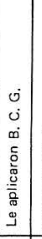 & 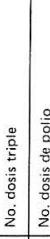 & 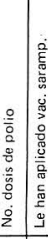 \\
\hline 1 & 2 & 3 & 4 & 6 & 7 & 8.5 & 10 & $11 !$ & & 14. & 15 & 16 . 17 & 18 & 19 & 20 & 21 & 2 & 2324 & 25 & 26 & \begin{tabular}{l|l}
27 & 28 \\
\end{tabular} & 29 & 30 & 31 & 32 & 33 & 3 & 35 & 36 & \begin{tabular}{l|l}
37 & 3 \\
\end{tabular} & 38 \\
\hline & & & & & & & & & & & & & & & & & & & & & & & & & & & & & & & \\
\hline & & & & & & & & & & & & & & & & & & & & & & & & & & & & & & & \\
\hline & & & & & & & & & & & & & & & & & & & & & & & & & & & & & & & \\
\hline & & & & & & & & & & & & & & & & & & & & & & & & & & & & & 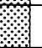 & & \\
\hline & & & & & & & & & & & & & & & & & & & & & & & & & & & & & & & \\
\hline & & & & & & & & & & & & & & & & & & & & & & & & & & & & & & & \\
\hline & & & & & & & & & & & & & & & & & & & & & & & & & & & & & & & \\
\hline & & & & & & & & & & & & & & & & & & & & & & & & & & & & & & & \\
\hline & & & & & & & & & & & & & & & & & & & & & & & & & & & & & & & \\
\hline & & & & & & & & & & & & & & & & & & & & & & & & & & & & & 8 & & \\
\hline & & & & & & & & & & & & & & & & & & & & & & & & & & & & & 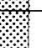 & & \\
\hline & & & & & & & & & & & & & & & & & & & & & & & & & & & & & & & \\
\hline & & & & & & & & & & & & & & & & & & & & & & & & & & & & & & & \\
\hline
\end{tabular}


4.9. Ulloa: Datos suministrados por la Promotora del Censo de Población y Vivienda de la localidad.

4.10. Andalucía: Listados de las promotoras de salud urbanas.

4.11. Restrepo: Oficina de Acuavalle.

4.12. Zarzal: Datos suministrados por las promotoras del censo de población y vivienda de la localidad.

4.13. Versalles: Datos suministrados trados por las promotoras de salud.

4.14. Sevilla: Secretaría de Salud, Censo de Vacunación, Oficina de Catastro y Listados de las Promotoras Urbanas.

4.15. La Cumbre: Listados de las promotoras de salud urbana.

4.16. Bolívar: Listados de las promotoras de salud urbana.

4.17. Caicedonia: Oficina de Acuavalle $y$ listados de las promotoras de salud urbana.

\section{CAPITULO VII}

\section{EL SUJETO}

\section{DELIMITACION}

Este estudio está dirigido hacia la población del subsector oficial del Sistema Nacional de Salud en el área geográfica del Departamento del Valle.

\subsection{Población asignada:}

Para efectos del presente estudio se considera población asignada el $73 \%$ de la población total del departamento del
Valle, teniendo en cuenta que la Seguridad Social atiende aproximadamente el $15 \%$ de la población y el sector privado el $12 \%$. Sin embargo, como consecuencia de la coordinación interinstitucional Servicio de Salud y Seguros Sociales, parte de los afiliados a la Seguridad Social son atendidos en los organismos del Sector Oficial; por esto en la etapa de programación este factor debe tenerse muy en cuenta.

1.2. Las Instituciones encargadas de atender a la población asignada al sector son:

- Hospital de máxima tecnología 1

- Hospitales especializados 2

- Hospitales regionales $\quad 10$

- Unidades intermedias 4

- Hospitales locales 34

- Centros de Salud Urbanos 29

- Puestos de Salud Rurales 264

- Zonas sanitarias 8

- Centros de Zoonosis 5

- Centro Regional de Adiestramiento

- Instituciones de Bienestar al anciano

\section{RECURSOS}

\subsection{Recursos financieros:}

Para funcionamiento en 1980 se contó un total de \$1.163.146.601.0o.

Recursos Financieros para Inversión: Un total de \$1.410.391.600.00

2.2. Recursos materiales:

Número de camas hospitalarias 
Número de consultorios médicos

Número de consultorios de enfermería

Número de consultorios odontológicos

Número de quirófanos.

\subsection{Recursos humanos:}

Número de médicos

* Número de odontólogos

Número de enfermeras

Número de otros profesionales

Número de auxiliares y ayudantes

Número de promotoras rurales

Número de promotoras urbanas

Número otro personal auxiliar

Número de promotores de saneamiento

Número personal administrativo

Número personal de servicios generales

\subsection{Recursos tecnológicos:}

Laboratorios cl ínicos

Laboratorios de patología

Laboratorios de rayos $X$

Unidades de rehabilitación

\footnotetext{
* Incluye las auxiliares de odontología social.
}

$453 \quad$ Unidades de cuidados intensivos

$85 \quad$ Unidades de cobalterapia y radioterapia

$94 \quad$ Unidades de terapia respiratoria

$52 \quad$ Unidades de cuidados especiales

2.5. Recursos legales

Decreto No. 056 de enero de 1975 Por el cual se sustituye el Decreto Ley No. 654 de 1974, y se dictan otras disposiciones.

\section{Decreto del Orden Nacional.}

Decreto No. 350 del 4 de marzo de 1975.

Por el cual se determina la organización y funcionamiento de los Servicios Seccionales de Salud y de las Unidades Regionales.

Decreto del Orden Nacional.

Decreto No. 356 del 5 de marzo de 1975.

Por el cual se establece el régimen de adscripción y vinculación de las entidades que presten servicios de salud.

Decreto del Orden Nacional.

Acuerdo No. 03 de julio 5 de 1976.

Por medio del cual se fija la organización y funcionamiento del Servicio Seccional de Salud del Valle del Cauca.

Acuerdo emanado de la Junta Seccional del Servicio de Salud del Valle.

Contrato de Constitución del Servicio Seccional de Salud del Valle del Cauca. 
17 de febrero de 1975, suscrito entre el Ministerio de Salud Pública y el Departamento del Valle para la integración del Servicio de Salud del Valle.

Convenio de Integración del Programa Control de Lepra al Servicio de Salud del Valle del Cauca.

Ley No. 09 del 24 de enero de 1979 (Código Sanitario Nacional). Por la cual se dictan medidas sanitarias.

Decreto No. 1210 de junio de 1978.

Por el cual se reglamenta el artículo 4o. de la Ley 14 de 1962 y el artículo 7o. del Decreto Ley 356 de 1975.

Con el fin de organizar las actividades docentes asistenciales en el Sistema $\mathrm{Na}$ cional de Salud.

Decreto del Orden Nacional.

Decreto Extraordinario No. 526 del 20 de marzo de 1975.

Por el cual se dictan normas sobre los Subsistemas Nacionales de Inversión, Información, Planeación, Suministros, Personal e Investigaciones del Sistema Nacional de Salud.

Expedido por el Presidente con base en las facultades extraordinarias, conferidas por la Ley 9a. de 1973 para reorganizar el Sistema Nacional de Salud y el Ministerio.

\section{ORGANIZACION Y ADMINISTRA- CION}

Los distintos recursos existentes se encuentran organizados en un sistema de prestación de servicios, cuyas características más importantes son:

- Estar constituido por una red de organismos de distinta complejidad.

- Estar coordinado con una jerarquización que consta de 3 niveles: Local, regional y central. Para una correcta funcionalidad y uso adecuado de recursos, está organizado por niveles de atención con un sistema de referencia.

Tanto la jerarquización como la atención por niveles está apoyada con una serie de elementos que le dan cohesión. Estos elementos son: políticas y normas que se ven reforzadas por la puesta en marcha de los subsistemas.

Todo esto dentro de un proceso de descentralización que permite una relación funcional entre los niveles se ve apoyada por la supervisión, asesoría y control, comunicación, transporte y educación.

La resultante de esto es la constitución de 42 niveles locales, agrupados en 9 unidades regionales, bajo el comando del nivel central del Servicio.

Funciones de los Niveles:

\section{Nivel Seccional:}

Adaptar la política nacional de salud a las características del departamento.

Elaborar el plan seccional y compatibilizarlo con la política nacional y analizar, compatibilizar y aprobar los programas y presupuestos de las unidades regionales de salud.

Asesorar, coordinar y supervisar las unidades regionales, y demás entidades públicas y privadas que actúan en el sector dentro de su jurisdicción, sobre el cumplimiento de las disposiciones establecidas en cuanto a prestación de los servicios, el ejercicio de las profesiones médicas y auxiliares, la producción, manejo y expendio de drogas y productos alimenticios, el funcionamiento de las instituciones y su administración.

Diseñar $y$ aplicar procesos que garanticen la formación, adiestramiento $y$ la 
actualización del personal y la administración $\mathrm{y}$ utilización racional de los recursos humanos.

Establecer mecanismos de coordinación entre las distintas entidades vinculadas del sector; $y$ de ellas con las otras vinculadas al mismo.

Establecer los mecanismos para la incorporación activa de todas las entidades adscritas y vinculadas de su jurisdicción a los subsistemas nacionales de planeación, inversión, suministros, investigación, personal y los demás que se establezcan.

Nivel Regional:

Elaborar programas específicos que en materia de salud se deban llevar a cabo a nivel regional, que estén en concordancia con la política nacional del sector, con los planes del Servicio y a partir de los proyectos de operación en las unidades locales, adaptándolas a las necesidades propias de la región.

Analizar, compatibilizar y aprobar los programas y presupuestos necesarios para llevar las actividades de las entidades adscritas y vinculadas a nivel local.

Asesorar, coordinar y supervisar la prestación de los servicios de salud en las unidades locales, de manera que respondan a las necesidades regionales, dentro de las normas técnicas y administrativas establecidas.

Realizar y evaluar programas de capacitación y administración de los recursos humanos del nivel regional, para garantizar el personal idóneo para un mejor servicio de salud a nivel local.

Aplicar a los organismos locales el régimen de adscripción y vinculación.

Instrumentar a nivel regional los subsistemas de información, planeación, in- versión, suministros, investigaciones, personal y los demás que se establezcan y promover la incorporación de las unidades locales a dichos subsistemas.

Nivel local:

Elaborar y ejecutar los programas de salud en el área de su jurisdicción, a partir de las propias necesidades y recursos, dentro de los delineamientos sobre prestación de servicios de atención a las personas y al medio, y sobre administración trazadas por los niveles superiores.

Someter a la aprobación del nivel regional los proyectos de programas de salud a desarrollar, para integrar las actividades de salud a los programas del nivel regional.

Elaborar el proyecto de presupuesto de la entidad y someterlo a revisión y aprobación del nivel regional correspondiente.

Prestar los servicios de medicina general, odontología y saneamiento básico en el área de su jurisdicción, conforme a los programas establecidos.

Contribuír a la capacitación y utilización del recurso humano, y a la educación de la comunidad en materia de salud.

Realizar los procedimientos que determinen los subsistemas nacionales, para activar los flujos de información y decisión en todo el sistema nacional de salud.

\section{CAPACIDAD DE OFERTA DE SERVICIOS}

Este sistema así organizado tiene una capacidad de oferta de servicios de acuerdo con la apertura programática en vigencia, a través de dos grandes progra- 
mas: atención a las personas y atención al ambiente, cada uno de ellos con las siguientes actividades finales:

Consulta médica: Se dispone de 441.416 horas/MD, que con un rendimiento optimizado de 4.4 consulta pueden generar 2.122.557 consultas que equivalen a consulta por persona asignada al año.

Hospitalización: Hay una dotación de 2.540 camas, que utilizadas con un grado de ocupación del $60 \%$ y con un promedio de días de estancia de 4.4. nos da capacidad para generar 125.682 egresos, lo cual, significa que por cada 17 personas asignadas hay capacidad de hospitalizar 1 al año.

Tratamiento odontológico: Se dispone de 163.000 horas adontológicas con un rendimiento de 4.0 tratamientos terminados por cada 8 horas, lo que nos da una capacidad de atención de 81.500 tratamientos lo que significa poder ofrecer un tratamiento terminado a 1 de cada 27 personas asignadas.

Control de enfermería: Hay una disponibilidad de 339.119 horas, que con un rendimiento de 4.5 controles/hora puede generar 1.541.291 controles, que para la población asignada de 783.725 nos da una disponibilidad de dos controles por personas al año.

Vacunación: El sistema posee capacidad para vacunar el $100 \%$ de la población asignada menor de 6 años.

Atención por promotora: La atención por promotora rural está dada por 266, que están en capacidad de atender a la totalidad de las familias asignadas, o sea las ubicadas en los núcleos poblacionales rurales con una concentración promedio de 5 visitas al año.

Promotoras urbanas: Existen 112 de ellas con una capacidad de atender 84.000 familias ubicadas en el área urbana con una concentración promedio de 4 visitas por año con un rendimiento de 2 visitas por hora.

Promotoras urbanas de salud y bienestar: Existen 30 en Cali, las cuales fuera de desempeñar funciones propias del Servicio de Salud, desempeñan también funciones asignadas por el ICBF. Tienen una capacidad para atender $20.190 \mathrm{fa}$ milias.

Atención al ambiente: Existen 360.565 horas promotor, lo que significa que se está en capacidad de atender el $64 \%$ de los sujetos existentes con la concentración y el rendimiento normalizados.

\section{DEMANDA}

La demanda está condicionada por una población de 2.208.078 habitantes distribuídas en 1.057.668 para hombres y 1.150 .408 para mujeres, distribuidos 485.777 en área rural y 1.722.299 en área urbana.

Enfrentamiento de la población al riesgo:

La población asignada al sector presenta una morbi-mortalidad por grupos de edad que se puede ver en la Tabla No. 15. Allí se presentan las principales causas tanto de morbilidad como de mortalidad, las cuales se caracterizan $y$ se enfrentan a las actividades finales con las cuales pueden atenderse.

Ubicación de la población.

Con base en la información tomada del documento editado por Planeación Departamental de 1980, los municipios del departamento están categorizados de acuerdo a la población urbana de la siguiente manera: 
Categoría I Municipios de más de200.000 habitantes-Cali.

Categoría II Municipios con población comprendida entre 101.000 y 200.000 habitantes: Buenaventura, $\mathrm{Pal}$ mira y Tuluá.

Categoría III Municipios con población comprendida entre 50.001 y 100.000 habitantes: Buga y Cartago.

Categoría IV Municipios con población entre 30.001 y 50.000 habitantes: Sevilla y Yumbo.

Categoría V Municipios con población entre 20.001 y 30.000 habitantes: Caicedonia, Cerrito, Florida y Zarzal.

Categoría VI Municipios con población rentre 10.001 y 20.000 habitantes: Candelaria, Guacarí, La Unión, Pradera, Roldanillo y Toro.

Categoría VII Municipios con población entre 5.001 y 10.000 habitantes: Alcalá, Andalucia, Ansermanuevo, Bugalagrande, Dagua, La Victoria, Obando y Trujillo.

Además incluímos los municipios de: Bolivar, Riofrio, Versalles, El Cairo, La Cumbre, El Aguila, Restrepo, Yotoco, Darién, San Pedro, Vijes, Ginebra, Ulloa, El Dovio y Argelia, cuya población urbana es menor de 5.000 habitantes.

De acuerdo con el censo sanitario rural, llevado a cabo por el Servicio de Salud en 1977, la población rural nucleada se encuentra distribuída en 969 veredas, las cuales fueron agrupadas en
267 unidades primarias. Además el departamento cuenta con un grupo de población dispersa la cual corresponde a un $7 \%$ total de la población.

El promedio de personas por vivienda es de 5.6, que está dentro del promedio nacional.

\subsection{Economía}

\section{Crecimiento económico:}

Con relación al producto bruto regional a precio de 1979 ha venido decreciendo en términos relativos siendo sus tasas las siguientes:

\begin{tabular}{|c|c|}
\hline Período & Aumento \% \\
\hline $1961-1966$ & 5.0 \\
$1966-1971$ & 6.5 \\
\hline $1971-1976$ & 3.0 \\
$1971-1977$ & 2.6 \\
$1971-1978$ & 2.9 \\
\hline
\end{tabular}

Los sectores de servicios durante el lapso 1971-1977 presentaron las siguientes tasas de crecimiento:

Transporte y comunicación

Electricidad y alquiler vivienda

Agricultura, caza, pesca y

silvicultura

Minería

Industria

Construcción

6.9 Educación:

Tomando como base el último pe- 
ríodo en 1978 la situación en el departamento es la siguiente:

\begin{tabular}{|l|c|r|c|}
\hline & Establecimientos & $\begin{array}{c}\text { Personal } \\
\text { docente }\end{array}$ & $\begin{array}{c}\text { Alumnos ma- } \\
\text { triculados }\end{array}$ \\
\hline Enseñanza primaria & 2.058 & 9.574 & 380.193 \\
Enseñanza media & 444 & 7.109 & 182.581 \\
(Oficial y privada) & 6 & 340 & 3.962 \\
Enseñanza intermedia & 8 & 1.616 & 19.143 \\
Enseñanza superior & & & \\
\hline
\end{tabular}

El porcentaje de analfabetismo es del $7 \%$.

\subsection{Seguridad Social.}

Cuenta el Valle del Cauca con las siguientes instituciones de Seguridad Social.

- Servicio médico del departamento "SEMEDE".

- Caja Nacional de Previsión

- Instituto de los Seguros Sociales

\section{Estudio Materno Infantil \\ Valle del Cauca 1980}

Tabla No. 1

\section{PROMEDIO DE PERSONAS \\ POR FAMILIA \\ SEGUN REGIONAL}

\begin{tabular}{|l|c|c|c|}
\hline \multirow{2}{*}{ Regional } & \multicolumn{2}{|c|}{ X personas X familia } & \multirow{2}{*}{ Total } \\
\cline { 2 - 3 } & Urbano & Rural & \\
\hline Cali & 5.2 & 4.4 & 5.2 \\
Palmira & 6.4 & 5.2 & 6.1 \\
Buga & 5.4 & 4.5 & 5.2 \\
Tulúa & 5.7 & 5.4 & 5.6 \\
Roldanillo & 5.0 & 6.1 & 5.5 \\
Cartago & 5.2 & 5.4 & 5.2 \\
Zarzal & 5.1 & 5.4 & 5.2 \\
Sevilla & 5.1 & 5.6 & 5.2 \\
B/ventura & 5.2 & - & 5.2 \\
\hline VALLE & 5.3 & 5.3 & 5.3 \\
\hline
\end{tabular}

Tabla No. 1. Se dá un promedio de $5.3 \%$ personas por familia para el Valle, teniendo como extremos a Palmira con $6.1 \%$; siendo sensiblemente igual para el área urbana y rural; es de resaltar el hecho de que el promedio de personas por familia en la década pasada presenta una tendencia a disminuír pasando de 6.2 a 5.3 en el período.

Tabla No. 2

\section{DISTRIBUCION PORCENTUAL DE LOS}

MENORES DE 1 AÑO

\begin{tabular}{|l|c|c|c|}
\hline \multirow{2}{*}{ Regional } & \multicolumn{2}{|c|}{$\begin{array}{r}\text { \% menores } \\
\text { de 1 año }\end{array}$} & \multirow{2}{*}{ Total } \\
\cline { 2 - 3 } & Urbano & Rural & \\
\hline Cali & 2.8 & 2.3 & 2.8 \\
Palmira & 3 & 2.8 & 3.2 \\
Buga & 3.0 & 2.6 & 2.9 \\
Tulúa & 2.5 & 2.9 & 2.7 \\
Roldanillo & 1.9 & 2.6 & 2.3 \\
Cartago & 3.9 & 4.3 & 3.9 \\
Zarzal & 2.6 & 2.3 & 2.4 \\
Sevilla & 2.7 & 2.6 & 2.7 \\
Buenaventura & 3.7 & - & 3.7 \\
\hline VALLE & 3.0 & 2.7 & 2.9 \\
\hline
\end{tabular}

Tabla No. 2. Para el total del Departamento es de $2.9 \%$ siendo los extre-mos Buenaventura con $3.7 \%$ y Zarzal con $2.4 \%$; siendo mayor este porcentaje en el área urbana que en la rural. En la década anterior este porcentaje ha ido en aumento pasando de 1.7 a 2.3 hasta llegar a $2.9 \%$ encontrado en el estudio.

Tabla No. 3. Para el total del Departamento se presenta el $24.7 \%$, extremos Buenaventura con $26.8 \%$ y Tulúa con $22.5 \%$. Este porcentaje ha ido en incremento pasando de un $22.4 \%$ a $24.7 \%$ encontrado. 
Tabla No. 3

PORCENTAJE DE MUJERES EN EDAD REPRODUCTIVA

\section{TASA GENERAL DE FECUNDIDAD $Y$ NATALIDAD $\left(10^{3}\right.$ HAB $)$}

\begin{tabular}{|l|c|c|c|}
\hline Regional & $\begin{array}{c}\% \text { g de } \\
15-44 \text { años } \\
\text { con respecto } \\
\text { a P. total }\end{array}$ & $\begin{array}{c}\text { Tasa } \\
\text { fecundidad } \\
\left(10^{3} \mathrm{~g}\right)\end{array}$ & $\begin{array}{c}\text { Tasa } \\
\text { de natalidad } \\
\left(10^{3} \text { hab }\right)\end{array}$ \\
\hline Cali & 24.7 & 9.3 & 21.5 \\
Palmira & 23.8 & 8.2 & 25.4 \\
Buga & 26.1 & 12.8 & 40.4 \\
Tuluá & 22.5 & 11.1 & 27.2 \\
Roldanillo & 25.7 & 9.7 & 22.6 \\
Cartago & 24.4 & 7.1 & 14.2 \\
Zarzal & 25.4 & 8.0 & 21.1 \\
Sevilla & 23.9 & 7.8 & 18.9 \\
Buenaventura & 26.8 & 5.7 & 22.1 \\
\hline VALLE & 24.7 & 8.9 & 22.1 \\
\hline
\end{tabular}

La tasa de fecundidad es del $8.9 \%$, siendos extremos Buga con $12.8 \%$ y Buenaventura con $5.7 \%$. Esta tasa en la década ha pasado de $7.5 \%$ a $8.9 \%$.

La tasa de natalidad encontrada fue de $22.1 \times 1.000$ siendo los extremos Buga con $40.4 \%$ y Cartago con $14.2 \%$. Esta tasa ha pasado de $16.8 \times 1.000$ a $17.4 \times 1.000$, hasta el 22.1 encontrado en el estudio.

Tabla No. 4. La tasa de mortalidad general es de $4.2 \times 1.000$, siendo los extremos Sevilla con 5.8 y Roldanillo con $1.5 \%$,

Esta mortalidad comparada con la del Departamento el año 1979, $5.0 \times 1.000$ es baja, puesto que la del año 1979 es sacada con base en los certificados de defunción; sin embargo, es posible que la diferencia se explique por un aumento en la mortalidad por accidentes de vehículo a motor, más frecuentemente utilizados por la clase media y alta.
Tabla No. 4

TASAS DE MORTALIDAD

GENERAL $\left(10^{3}\right.$ HAB)

INFANTIL $\left(10^{3}\right.$ MENORES)

$Y$ MORTALIDAD

PROPORCIONAL (\%) DE

MENORES DE UN

AÑO CON RESPECTO AL

TOTAL DE DEFUNCIONES

\begin{tabular}{|l|c|c|c|}
\hline Regional & $\begin{array}{c}\text { Mortalidad } \\
\text { general } \\
\left(10^{3} \text { HAB }\right)\end{array}$ & $\begin{array}{c}\text { Mortalidad } \\
\text { infantil } \\
\left(10^{3} \text { N.V. }\right)\end{array}$ & $\begin{array}{c}\% \\
\text { Muertos en } \\
1 \text { año }\end{array}$ \\
\hline Cali & 4.7 & 52.4 & 23.9 \\
Palmira & 3.8 & 57.3 & 37.9 \\
Buga & 4.8 & 46.4 & 38.9 \\
Tuluá & 4.6 & 75.3 & 44.0 \\
Roldanillo & 1.5 & 50.8 & 75.0 \\
Cartago & 1.7 & 54.5 & 46.2 \\
Zarzal & 2.2 & 51.3 & 50.0 \\
Sevilla & 5.8 & 76.5 & 25.0 \\
Buenaventura & 5.6 & 73.2 & 28.6 \\
\hline VALLE & 4.2 & 57.4 & 29.8 \\
\hline
\end{tabular}

La mortalidad infantil para el Departamento dió $57.4 \times 1.000$, siendo los extremos Sevilla con $76.5 \%$ y Buga con $46.4 \%$. Esta mortalidad no es comparable con la del Departamento; en 1979 fue de $39.9 \times 1.000$ puesto que esta es una tasa por mil habitantes mientras que la del estudio es por mil nacidos vivos.

El porcentaje de muertes en menores de 1 año fue del $29.8 \%$, siendo los extremos Roldanillo con $75 \%$ y Cali con el $23.9 \%$. Para 1979 la del Departamento fue del $18 \%$.

Tabla No. 5. El estudio por primera vez está discriminado por grupos de 5 años; el mayor porcentaje $34.1 \%$ corresponde al grupo de 20-24 años, 
Tabla No. 5

DISTRIBUCION PORCENTUAL POR GRUPOS DE EDAD (AÑOS) DE LOS GESTANTES

\begin{tabular}{|l|c|c|c|c|c|c|c|c|}
\hline \multirow{2}{*}{ Regional } & \multicolumn{7}{|c|}{ Grupos de Edad (Años) } & \multirow{2}{*}{ Total } \\
\cline { 2 - 9 } & $15-19$ & $20-24$ & $25-29$ & $30-34$ & $35-39$ & $40-44$ & 45 y + & \\
\hline Cali & 23.2 & 34.9 & 20.9 & $10 / .8$ & 7.5 & 2.2 & .6 & 100.0 \\
Palmira & 16.4 & 36.0 & 27.0 & 11.6 & 5.8 & 3.2 & .0 & 100.0 \\
Buga & 22.0 & 35.0 & 25.2 & 12.2 & 4.0 & 1.6 & .0 & 100.0 \\
Tuluá & 13.4 & 31.6 & 18.8 & 19.5 & 11.4 & 4.0 & 1.3 & 100.0 \\
Roldanillo & 12.7 & 33.3 & 25.4 & 15.9 & 9.5 & 3.2 & .0 & 100.0 \\
Cartago & 9.0 & 29.5 & 32.8 & 9.9 & 11.5 & 5.7 & 1.6 & 100.0 \\
Zarzal & 13.7 & 31.5 & 26.7 & 15.8 & 9.6 & 2.1 & .7 & 100.0 \\
Sevilla & 10.5 & 34.2 & 23.2 & 19.5 & 8.4 & 2.6 & 1.6 & 100.0 \\
Buenaventura & 26.7 & 36.7 & 21.6 & 10.0 & 5.0 & .0 & .0 & 100.0 \\
\hline VALLE & 18.5 & 34.1 & 23.2 & 13.0 & 7.9 & 2.6 & .7 & 100.0 \\
\hline
\end{tabular}

además existe el grupo de 15-19 años equivalente al $18.5 \%$ y los grupos por encima de los 40 años que llegan al

Tabla No. 6

PROMEDIO DE PARTOS

\begin{tabular}{|c|c|c|}
\hline Regional & $\bar{x}$ & Partos \\
\hline Cali & & 2.6 \\
\hline Palmira & & 2.8 \\
\hline Buga & & 2.6 \\
\hline Tuluá & & 3.5 \\
\hline Roldanillo & & 2.6 \\
\hline Cartago & & 3.5 \\
\hline Zarzal & & 2.4 \\
\hline Sevilla & & 3.5 \\
\hline Buenaventura & & 1.4 \\
\hline VALLE & & 2.8 \\
\hline
\end{tabular}

$3.3 \%$, grupos éstos denominados de alto riesgo, a los cuales hay necesidad de darles mayor atención.

Tabla No. 6. Para el Valle es de $2.8 \%$, siendo los extremos Sevilla, Cartago y Tuluá con $3.5 \%$ y Buenaventura con $1.4 \%$.

Tabla No. 7. Para el total en el Valle en el 1er. trimestre tuvieron control $52.6 \%$, en el 2 do. trimestre el $67.6 \%$ $y$ en el 3er. trimestre el $70.9 \%$. Tendencia esta que corrobora la impresión subjetiva de la forma tardía en que la mujer solicita control cuando está en embarazo.

Es relevante mencionar que un $6.2 \%$ nunca asiste a control teniendo la prestación del servicio para que lo hiciera.

Tabla No. 8. Es de anotar como sólo el $5.8 \%$ llevan control en la casa, a nivel de médico particular se encontró 
Tabla No. 7

\section{$\%$ GESTANTES QUE FUERON A CONTROL PRENATAL}

\section{SEGUN TRIMESTRE DE GESTACION}

\begin{tabular}{|l|c|c|c|c|}
\hline \multirow{2}{*}{ Regional } & \multicolumn{2}{|c|}{ \% control según trimestre } & \multirow{2}{*}{$\begin{array}{c}\text { \% nunca asistie- } \\
\text { ron a rontrol }\end{array}$} \\
\cline { 2 - 4 } & $\mathrm{I}$ & $\mathrm{II}$ & $\mathrm{III}$ & \\
\hline Cali & 56.1 & 76.4 & 81.5 & 6.8 \\
Palmira & 38.8 & 57.1 & 59.5 & 6.3 \\
Buga & 64.3 & 70.3 & 77.7 & 10.1 \\
Tuluá & 43.4 & 48.0 & 51.3 & 3.3 \\
Roldanillo & 72.7 & 76.9 & 33.3 & 1.5 \\
Cartago & 43.9 & 56.8 & 56.1 & 3.1 \\
Zarzal & 46.5 & 75.6 & 74.3 & 9.7 \\
Sevilla & 48.7 & 60.2 & 36.7 & 4.7 \\
Buenaventura & 59.0 & 54.0 & 550 & 4.3 \\
\hline \multicolumn{1}{|c|}{ VALLE } & 52.6 & 67.6 & 70.9 & 6.2 \\
\hline
\end{tabular}

el $7.1 \%$, el resto el $81.5 \%$ llevan control institucional.

Tabla No. 9. El 5\% fue controlado, por partera que coincide con el $5.8 \%$ de control en la casa; por médico se realizó el $47.8 \%$, por auxiliar de enfermería el $42 \%$, por enfermera el $7.4 \%$.

Tabla No. 10. El $89.4 \%$ termina en forma normal, el $6 \%$ termina en césarea, un $2.2 \%$ en parto prematuro y un $2.4 \%$ en aborto.

El porcentaje de césareas comparado con el atendido a nivel de clínicas es menor.

Tabla No. 11. Sólo el $25.8 \%$ fue atendido en casa, el resto $(71.5 \%)$ tuvo atención institucional, dato este que concuerda con el análisis de causas de egresos para el Departamento, cuya primera causa es parto normal.

Tabla No. 12. Sólo el $21.2 \%$ fue atendido por partera, el resto $78.8 \%$ fue atendido por personal médico o

Tabla No. 8

\section{DISTRIBUCION PORCENTUAL SEGUN LUGAR DEL CONTROL PRENATAL - VALLE}

\begin{tabular}{|l|r|r|r|r|r|r|r|}
\hline \multicolumn{7}{|c|}{ Lugar del Control Prenatal (\%) } \\
\hline \multicolumn{1}{|c|}{ Regional } & $\begin{array}{c}\text { Centro de } \\
\text { Salud }\end{array}$ & I. S. S. & Hospital & $\begin{array}{c}\text { Médico } \\
\text { Particular }\end{array}$ & Casa & Otros & Total \\
\hline Cali & 53.4 & 24.5 & 10.7 & 4.9 & 3.4 & 3.1 & 100.0 \\
Palmira & 13.3 & 31.1 & 34.1 & 5.2 & 4.4 & 11.9 & 100.0 \\
Buga & 8.0 & 26.0 & 50.0 & 6.0 & 7.0 & 3.0 & 100.0 \\
Tuluá & 40.6 & 14.3 & 26.4 & 9.9 & 3.3 & 5.5 & 100.0 \\
Roldanillo & 42.6 & 6.4 & 29.8 & 12.8 & 4.2 & 4.2 & 100.0 \\
Cartago & 5.4 & 16.2 & 55.4 & 9.5 & 2.7 & 10.8 & 100.0 \\
Zarzal & 16.2 & 25.6 & 41.9 & 9.4 & 2.5 & 3.4 & 100.0 \\
Sevilla & 13.1 & 0.0 & 49.0 & 8.3 & 17.2 & 12.4 & 100.0 \\
Buenaventura & 7.2 & 4.8 & 47.0 & 16.9 & 19.3 & 4.8 & 100.0 \\
\hline \multicolumn{1}{|c|}{ VALLE } & 34.0 & 20.3 & 27.2 & 7.1 & 5.8 & 5.6 & 100.0 \\
\hline
\end{tabular}


Tabla No. 9

PERSONAL ENCARGADO DEL CONTROL PRENATAL

\begin{tabular}{|c|c|c|c|c|c|c|c|}
\hline \multicolumn{7}{|c|}{ Personal encargado del control prenatal (\%) } \\
\hline Regional & Médico & Auxiliar & Enfermera & Partera & $\begin{array}{c}\text { M. D. Enf. } \\
\text { Auxiliar }\end{array}$ & $\begin{array}{c}\text { Enf. y } \\
\text { Aux. }\end{array}$ & Total \\
\hline VALLE & 47.8 & 22.0 & 7.4 & 5.0 & 16.6 & 42 & 100.0 \\
\hline
\end{tabular}

- Intervalo de confianza $(95 \%)$ para atención por médico $<50.2 \%$

Tabla No. 10

FORMA DE TERMINACION DEL EMBARAZO

\begin{tabular}{|l|c|c|c|c|c|c|c|}
\hline \multicolumn{1}{|c|}{ Regional } & Normal & Cesárea & $\begin{array}{c}\text { Parto } \\
\text { Prematuro }\end{array}$ & $\begin{array}{c}\text { Aborto } \\
\text { I. Trim. }\end{array}$ & $\begin{array}{c}\text { Aborto } \\
\text { II. Trim. }\end{array}$ & Otras & Total \\
\hline Cali & 88,1 & 7.5 & 2.6 & .6 & 1.0 & .2 & 100.0 \\
Palmira & 87.8 & 6.9 & 2.1 & 2.1 & 1.1 & .0 & 100.0 \\
Buga & 92.8 & 3.6 & 2.9 & .0 & .7 & .0 & 100.0 \\
Tuluá & 89.4 & 5.3 & .0 & 3.3 & 2.0 & .0 & 100.0 \\
Roldanillo & 89.2 & 6.2 & .0 & 3.1 & 1.5 & .0 & 100.0 \\
Cartago & 93.8 & 3.8 & .8 & .8 & .8 & .0 & 100.0 \\
Zarzal & 84.8 & 9.0 & 3.4 & 1.4 & 1.4 & .0 & 100.0 \\
Sevilla & 92.6 & 1.1 & 2.1 & 2.6 & 1.6 & .0 & 100.0 \\
Buenaventura & 95.8 & 2.1 & 2.1 & .0 & .0 & .0 & 100.0 \\
\hline \multicolumn{1}{|c|}{ VALLE } & 89.4 & 6.0 & 2.2 & 1.2 & 1.0 & .2 & 100.0 \\
\hline
\end{tabular}

- Límites de confianza $\left(95 \%\right.$ ) para Parto Normal $<\begin{array}{r}90.8 \% \\ 88.0 \%\end{array}$

paramédico. El dato de $21.2 \%$ es consistente con el de $25.8 \%$ de lugar donde se atendió el parto que corresponde en la casa.
Tabla No. 13. El $95.9 \%$ fue tipo único $y$ el $4.1 \%$ múltiple.

Tabla No. 14. El 81.1\% no presentó 
Tabla No. 11

LUGAR DE ATENCION DEL PARTO $(\%)$

\begin{tabular}{|c|c|c|c|c|c|c|c|}
\hline Regional & Hospital & I.S.S. & Casa & $\begin{array}{l}\text { Centro } \\
\text { de Salud }\end{array}$ & $\begin{array}{l}\text { Clínica } \\
\text { Particular }\end{array}$ & Otros & Total \\
\hline Cali & 28.2 & 23.1 & 21.1 & 23.0 & 3.8 & .8 & 100.0 \\
\hline Palmira & 49.3 & 26.0 & 17.3 & 4.7 & 2.7 & .0 & 100.0 \\
\hline Buga & 56.5 & 22.6 & 17.4 & 1.7 & .9 & .9 & 100.0 \\
\hline Tuluá & 28.1 & 12.4 & 39.7 & 19.8 & .0 & .0 & 100.0 \\
\hline Roldanillo & 63.0 & 5.6 & 27.8 & 1.8 & 1.8 & .0 & 100.0 \\
\hline Cartago & 42.1 & 15.0 & 34.6 & 3.7 & 3.7 & .9 & 100.0 \\
\hline Zarzal & 57.7 & 16.8 & 21.1 & 2.2 & 2.2 & .0 & 100.0 \\
\hline Sevilla & 57.0 & .6 & 38.5 & 2.8 & .0 & .0 & 100.0 \\
\hline Buenaventura & 45.9 & 2.3 & 44.7 & 2.4 & 4.7 & 1.1 & 100.0 \\
\hline VALLE & 39.8 & 17.7 & 25.8 & 13.3 & 2.7 & .7 & 100.0 \\
\hline
\end{tabular}

- Intervalo de confianza $(95 \%)$ para atención Parto en casa $<23.8 \%$

Tabla No. 12

PERSONA ENCARGADA DE LA ATENCION DEL PARTO (\%)

\begin{tabular}{|c|c|c|c|c|c|c|c|}
\hline Regional & Médico & Partera & Auxiliar & Enfermera & $\begin{array}{c}\text { M.D. } \\
\text { Aux. Enf. }\end{array}$ & Otras & Total \\
\hline Valle & 49.8 & 21.2 & 13.8 & 10.0 & 1.7 & 3.5 & 100.0 \\
\hline
\end{tabular}

complicación, el $9.2 \%$ presentó hemorragia, el $2.8 \%$ desgarro, el $3.6 \%$ fiebre hasta por 8 días.

Es de resaltar que el $92.5 \%$ de las gestantes presentó parto a término.

Tabla No. 15. El $84 \%$ no presentó complicación consistente, con el $81 \%$ de madres que no presentaron compli- cación en el momento del parto, que presentaron complicación. Porcentaje este muy similar a los grupos de atención de alto riesgo.

$51.2 \%$ de las gestantes consultaron después del parto o aborto, porcentaje este considerado como bajo, lo hacen a instituciones oficiales. 
Tabla No. 13

PORCENTAJE DE GESTANTES SEGUN TIPO DE PARTO

\begin{tabular}{|l|r|r|r|}
\hline \multicolumn{1}{|c|}{ Regional } & Unico & Multiple & Total \\
\hline Cali & 98.4 & 1.6 & 100.0 \\
Palmira & 89.4 & 10.6 & 100.0 \\
Buga & 94.7 & 5.3 & 100.0 \\
Tuluá & 99.3 & .7 & 100.0 \\
Roldanillo & 98.3 & 1.7 & 100.0 \\
Cartago & 95.9 & 4.1 & 100.0 \\
Zarzal & 99.3 & .7 & 100.0 \\
Sevilla & 98.9 & 1.1 & 100.0 \\
Buenaventura & 67.0 & 33.0 & 100.0 \\
\hline \multicolumn{1}{|c|}{ VALLE } & 95.9 & 4.1 & 100.0 \\
\hline
\end{tabular}

VACUNACION

Coberturas alcanzadas en Vacunación 1981

\begin{tabular}{|ll|}
\hline \multicolumn{2}{|c|}{ Menores de 1 año: } \\
\hline BCG & $82 \%$ \\
DPT & $30.95 \%$ \\
POLIO & $33.4 \%$ \\
SARAMPION & $36.7 \%$ \\
\hline EMBARAZADAS TT $19.3 \%$ \\
\hline
\end{tabular}

Límites de confianza $(95 \%)$ para Parto Unico $<\begin{array}{r}96.8 \% \\ 95.0 \%\end{array}$

Tabla No. 14

\section{DISTRIBUCION PORCENTUAL SEGUN TIPO DE COMPLICACION} MATERNA DURANTE EL PARTO O ABORTO

\begin{tabular}{|l|r|r|r|r|c|c|c|}
\hline \multicolumn{7}{|c|}{ Tipo de complicación materna } \\
\hline Regional & Ninguna & $\begin{array}{c}\text { Durante } \\
\text { Parto }\end{array}$ & $\begin{array}{r}\text { Post- } \\
\text { Parto }\end{array}$ & Desgarres & $\begin{array}{c}\text { Fiebre has- } \\
\text { ta 8 días }\end{array}$ & Otras & Total \\
\hline Cali & 83.2 & 4.5 & 3.2 & 2.7 & 3.6 & 2.8 & 100.0 \\
Palmira & 72.2 & 3.2 & 5.2 & 5.2 & 7.1 & 7.1 & 100.0 \\
Buga & 80.0 & 11.3 & .9 & 1.7 & 4.4 & 1.7 & 100.0 \\
Tuluá & 83.7 & 6.4 & 6.4 & 1.4 & .7 & 1.4 & 100.0 \\
Roldanillo & 65.4 & 5.5 & 10.9 & 1.8 & 7.3 & 9.1 & 100.0 \\
Cartago & 83.4 & .9 & 4.6 & .9 & 1.9 & 8.3 & 100.0 \\
Zarzal & 81.7 & 5.8 & 1.5 & 5.1 & 4.4 & 1.5 & 100.0 \\
Sevilla & 80.6 & 5.0 & 8.3 & 1.7 & 2.2 & 2.2 & 100.0 \\
Buenaventura & 83.5 & 3.5 & 4.7 & 4.7 & 2.4 & 1.2 & 100.0 \\
\hline \multicolumn{1}{|c|}{ VALLE } & 81.1 & 4.9 & 4.3 & 2.8 & 3.6 & 3.3 & 100.0 \\
\hline
\end{tabular}

Intervalo de confianza $(95 \%)$ para ninguna complicación $<-82.9 \%$ 
Tabla No. 15

\section{DISTRIBUCION PORCENTUAL SEGUN TIPO DE COMPLICACION DEL NIÑO $(\%)$}

\begin{tabular}{|c|c|c|c|c|c|c|}
\hline Regional & Ninguna & $\begin{array}{c}\text { Demora en } \\
\text { respirar }\end{array}$ & Morado & $\begin{array}{c}\text { Fue hospita- } \\
\text { lizado }\end{array}$ & Otras & Total \\
\hline VALLE & 84.0 & 5.3 & 5.1 & 2.0 & 3.6 & 100.0 \\
\hline
\end{tabular}

- Límite de confianza $(95 \%)$ para ninguna complicación $<-85.7 \%$

Tabla No. 16

TASA DE MORTALIDAD X 1000 N.V.

\begin{tabular}{|l|c|r|r|r|r|}
\hline \multicolumn{7}{|c|}{ Tasas de mortalidad $\left(10^{3}\right.$ N.V.) } \\
\hline Regional & $\begin{array}{c}\text { Al } \\
\text { Nacimiento }\end{array}$ & 8 días & $8-23$ días & Perinatal & Neonatal \\
\hline Cali & 29.1 & 8.2 & 5.8 & 37.3 & 14 \\
Palmira & 62.5 & 5.2 & 26.0 & 67.7 & 31.2 \\
Buga & 19.9 & 33.1 & 0.0 & 53.0 & 33.1 \\
Tuluá & 21.7 & .0 & .0 & 21.7 & .0 \\
Roldanillo & 50.8 & .0 & .0 & 50.8 & .0 \\
Cartago & 18.2 & 27.3 & .0 & 45.5 & 27.3 \\
Zarzal & 51.3 & 42.7 & .0 & 94.0 & 42.7 \\
Sevilla & 49.2 & 10.9 & .0 & 60.1 & 10.9 \\
Buenaventura & 61.0 & 36.6 & .0 & 97.6 & 36.6 \\
\hline \multicolumn{1}{|c|}{ TOTAL } & 34.7 & 13.7 & 5.3 & 48.4 & 19.0 \\
\hline Internado de & 42.9 & 18.9 & 8.6 & & \\
confianza & 26.5 & 8.5 & 2.0 & & \\
del 95\% & \multicolumn{5}{|l}{} \\
\hline
\end{tabular}

Tabla No. 16. Para el Valle fue del $34.7 \%$ por mil nacidos vivos, siendo los extremos Palmira con el $62.5 \% \times 1.000$ y Cartago con el $82.4 \times 1.000$ 
La mortalidad en menores de 8 dias fue de $1.37 \times 1.000$, la mortalidad entre los 8 y 28 días fue del $5.3 \times 1.000$ la mortalidad neonatal fue del $19 \times$ 1.000 .

\section{CAPITULO 8}

\section{Resumen para Cali}

Tiene una mayor proporción de mujeres en edad reproductiva, que precozmente se embarazan pero que limitan su fecundidad probablemente con métodos anticonceptivos efectivos. Al embarazarse asisten en su mayoría a control prenatal $\mathrm{y}$ atención del parto a instituciones oficiales pero aún el $20 \%$ tienen su parto en casa.

En el $14 \%$ se complica el niño al nacer y la madre en el $17 \%$ de los casos.

Sólo la mitad asisten al control postparto o aborto y llevan al niño a consulta de niño sano.

La mortalidad perinatal está dada en gran proporción por los mortinatos que es necesario estudiar para aclarar sus causas.

La mortalidad infantil aún es alta.

\section{MATERNAL AND CHILD CARE SUMMARY FOR CALI}

There is a greater proportion of women in fertile years that become pregnant at an early age in this city; they probably limit their fertility through effective contraception. Most of them seek antenatal care and go to public hospitals for delivery, but there is still $20 \%$ who give birth at home.

In $14 \%$ of the cases there are com- plications of the newborn and in $17 \%$ there are complications of the mother.

Only $50 \%$ attend postpartum follow-up or postabortion check-ups and take the child to the routine medical visit.

Perinatal mortality is constitued by stillbirths whose causes must be carefully studies. Infant mortality is quite high.

\section{MATERNAL AND CHILD HEALTH CARE - COMAPARTIVE STUDY}

\section{JANUARY - DECEMBER, 1980}

\section{Conclusions and recommendations}

1. Once the basic diagnosis is made, it seems convenient to bring some light on the differences found in the indicators of maternal and child health both in the cities and the health regions studied.

2. It is advisable to introduce as a rule a system of surveillance on the mother and child population in order to obtain accurate information about the diverse health indicators.

3. A close coordination between the public sector and scientific associations should be prometed, so that the latter may participate in the design and implementation of a set of norms for the prevention and provision of services to mother and child. 
4. The health indicators that are specific to each region should be considered, since the national or state averages may not reflect the exact health or disease situation.

5. Research on mother and child health must be broadened so that a better insight can be used in takin correc-

\section{Bibliografía}

1. Administración de Salud Pública, texto Dr. Roberto Hernández Elías, profesor en la Unidad Docente Nacional "C.J. Finlay" Ciencia y Técnica. Instituto $\mathrm{Cu}$ bano del Libro. La Habana 1971.

2. Factores Condicionantes de la Salud del Niño. Doctores: Carlos Hernán Daza y Mario Eisler. Condiciones de Salud del Niño en las Américas O.P.S. 1979 Publicación Científica No. 381. Págs. 57-80.

3. Las Condiciones de Salud en las Américas 1969-1972. Publicación Científica de la OPS 287 Washington D.C. 1974.

4. Programas para mejorar la nutrición de las mujeres embarazadas y madres lactantes. Doctores: Carlos Hernán Daza y Aaron Lechting. Bol. OPS Vol. 89 No. 6 Dic. 1980 573-587.

5. Guía para interpretar la ganancia de peso durante el embarazo como indicador de riesgo de bajo peso al nacer. Doctores: Aaron Lechting y Robert. E. Klein. Of. Sanit. Panam. 89 (6) 1980. 489-493.

6. Características de la morbimortalidad en el adolescente $y$ el joven. Doctores: Marco Cusminsky y Elbio Néstor Suárez Ojeda. Condiciones de Salud del niño en las Américas OPS 1979 Publicación Científica 38 , págs. 36-56.

7. Atención Materno-Infantil. Doctora: Sonia Pazmiño de Osorio. Revista Colom- tive measures for public sector programs.

6. Given the different stages of development of this country's political and administrative sections, it is urgent to establish specific diagnosis for each region and conduct further research according to initial findings.

biana de Obstetricia y Ginecología. Vol. XXXI No. 2 pág. 80. Marzo-Abril 1980.

8. Puffer, R.R. y C.V. Serrano. Características de la mortalidad en el niño. Publicación Científica de la OPS 262, Washington, D.C. 1973.

9. Salud Ocupacional en América Latina y el Caribe. Consideraciones sobre algunos problemas, alternativas, tendencias $y$ desafíos para su promoción. OPS. VI Congreso Interamericano de Prevención de Riesgos Profesionales, Caracas. Venezuela X. 1981.

10. Ministerio de Salud Pública de Cuba O.P.S. O.M.S. Taller Internacional sobre metodología para la normalización de la atención Materno-Infantil, 20, 23, Nov. 1979. La Habana, Cuba.

11. Método de Atención Sanitaria de la madre y el niño, basado en el concepto de riesgo, OMS publicación en offset No. 39, Ginebra 1978.

12. Estudio de los factores de riesgo obstétrico en las gestantes cubanas. Ministerio de Salud Pública de Cuba, 1980.

13. R.R.: Puffer y C.V. Serrano "Results of the Inter-American Investigations ofmortality relating to reproduction". En epidemology of abortion and practices of fertility regulation in Latin America: selected reports (PAHO Publications CientificiNo. 306. 1975. 
14. Agualimpia Carlos. Investigación Nacional de Morbilidad Estudio de Recursos Humanos para la Salud y Educación Médica en Colombia, 1964.

15. Evaluación del Programa Materno-Infantil de Colombia 1974-1976 Julio 1976. Ministerio de Salud OPS, Fondo de las Naciones Unidas para Actividades de Población.

16. Servicio de Salud del Valle del Cauca. Evaluación del programa de Atención Materno-Infantil. 1975.

17. Ministerio de Salud. Boletín Epidemiológico Nacional. Julio-Septiembre 1977. Vol. 3 No. 3-4.

18. Joe D. Wray y Alfredo Aguirre, "Protein Calorie Malnutrition in Candelaria, Colombia. Prevalence, Social and Demographic Causal Factor" Journal of Tropical Pediatrics 15 (1969): 91, 93, 97.
19. Susan Scrimshawetal, “Aceptabilidad e Impacto: La Evaluación de un Programa Modelo de Prestación de Servicios de Salud en Cali, Colombia, Nueva York: Universidad de Columbia, Instituto Internacional para el estudio de la reproducción humana. 1975.

20. Susan Ch. Scrimshawetal, “La mortalidad infantil $y$ el comportamiento respecto a la regulación del tamaño de la familia. Estudios de población. Número especial abril de 1980. pág. 81-93.

21. Departamento Nacional de Planeación, Plan de Integración Nacional 1979-1982 Tomo 1 y 2, 1a. ed. mayo 1980.

22. Instituto Nacional de Nutrición. Fundamentos de Medicina Hernán Velez, Jaime Borrero, Jorge Restrepo Molina, texto Universidad de Antioquia. pág. 5.

23. Financiera Minsalud. 